OPEN ACCESS

Edited by: Jagadeesh Bayry,

Institut National de la Santé et de la Recherche Médicale

(INSERM), France

Reviewed by: Kithiganahalli Narayanaswamy Balaji, Indian Institute of Science, India Min Wu,

University of North Dakota, United States

*Correspondence:

Mrigank Srivastava mrigank_srivastava@cdri.res.in

Specialty section:

This article was submitted to

Molecular Innate Immunity,

a section of the journal

Frontiers in Immunology

Received: 26 August 2017

Accepted: 23 January 2018

Published: 12 February 2018

Citation:

Sharma A, Sharma P, Ganga L,

Satoeya N, Mishra S,

Vishwakarma AL and Srivastava M (2018) Infective Larvae of Brugia malayi Induce Polarization of Host

Macrophages that Helps in Immune Evasion.

Front. Immunol. 9:194. doi: 10.3389/fimmu.2018.00194

\section{Infective Larvae of Brugia malayi Induce Polarization of Host Macrophages that Helps in Immune Evasion}

\author{
Aditi Sharma ${ }^{1,2}$, Pankaj Sharma', Laxmi Ganga ${ }^{1}$, Neha Satoeya', Shikha Mishra', \\ Achchhe Lal Vishwakarma ${ }^{3}$ and Mrigank Srivastava ${ }^{1,2 *}$
}

${ }^{1}$ Parasitology Division, CSIR-Central Drug Research Institute, Lucknow, India, ${ }^{2}$ Academy of Scientific and Innovative Research (AcSIR), New Delhi, India, ${ }^{3}$ Sophisticated Analytical Instrument Facility (SAIF), CSIR-Central Drug Research Institute, Lucknow, India

Filarial parasites suppress, divert, or polarize the host immune response to aid their survival. However, mechanisms that govern the polarization of host MФs during early filarial infection are not completely understood. In this study, we infected BALB/c mice with infective larvae stage-3 of Brugia malayi (Bm-L3) and studied their effect on the

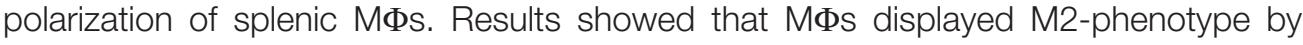
day 3 p.i. characterized by upregulated IL-4, but reduced IL-12 and Prostaglandin-D2 secretion. Increased arginase activity, higher arginase-1 but reduced NOS2 expression and poor phagocytic and antigen processing capacity was also observed. M2 MФs supported T-cell proliferation and characteristically upregulated p-ERK but downregulated NF-kB-p65 and NF-кB-p50/105. Notably, Bm-L3 synergized with host regulatory T-cells

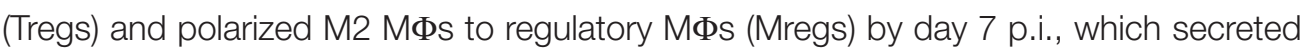
copious amounts of IL-10 and prostaglandin-E2. Mregs also showed upregulated expression levels of MHC-II, CD80, and CD86 and exhibited increased antigen-processing capacity but displayed impaired activation of NF-kB-p65 and NF-kB-p50/105. Neutralization of Tregs by anti-GITR + anti-CD25 antibodies checked the polarization of M2 MФs to Mregs, decreased accumulation of regulatory B cells and inflammatory monocytes, and reduced secretion of IL-10, but enhanced IL-4 production and percentages of eosinophils, which led to Bm-L3 killing. In summary, we report hitherto undocumented effects of early Bm-L3 infection on the polarization of splenic MФs and show how infective larvae deftly utilize the functional plasticity of host MФs to establish themselves inside the host.

\section{Keywords: filariasis, polarization, $\mathrm{M} 2 \mathrm{M \Phi}$, regulatory $\mathrm{M \Phi}$, regulatory T-cell, regulatory B-cell, MAP kinase}

\section{INTRODUCTION}

MФs are sentinel immune cells that are centrally involved in antimicrobial defense, tissue repair, clearing cell debris, and maintaining homeostasis. Two major populations of MФs have been identified and characterized in the mammalian system. M1 MФs, also known as classically activated macrophages are induced by Th1 cytokine, IFN- $\gamma$, and exhibit a proinflammatory profile while M2

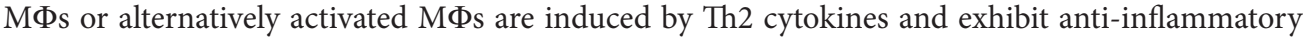
activities and tissue-repair functions $(1,2)$. In an in vitro setting, three different phenotypes of 
bone marrow-derived $M \Phi(B M M \Phi)$ have been described based on whether these cells were primed with IFN- $\gamma$, LPS, immune complexes, or IL-4 (3). However, during in vivo conditions, MФs being plastic in nature adapt to the surrounding stimuli and rapidly change their phenotype. In fact, it is this process of $M \Phi$ polarization that essentially regulates and decides the ultimate fate of the host immune response.

Filarial parasites stimulate the induction of M2 MФs and impart profound functional changes in antigen-presenting cells viz. Dendritic cells (DCs) and M $\Phi$ s that lead to an impaired Th1, but dominant Th2 immune response that provide protection during parasitic infections $(4,5)$. In addition to this, asymptomatic individuals harbor another phenotype of MФs known as the regulatory MФs (Mregs), which are characterized by high amounts of IL-10 that lead to modified type 2 responses and contribute to enhanced parasite survival. We also recently reported functional impairment of host DC subsets and attenuated T-cell response during early Bm-L3 infection (6). However, mechanisms that regulate the polarization of host $\mathrm{M} \Phi$ s following $\mathrm{Bm}-\mathrm{L} 3$ infection remain unanswered. In the present study, we infected $\mathrm{BALB} / \mathrm{c}$ mice with Bm-L3, and monitored the polarization of splenic $M \Phi$ s during the first week of infection. We observed alternatively activated phenotype of splenic $\mathrm{M} \Phi$ s at day 3 p.i., which rapidly changed to a regulatory phenotype at day 7 p.i.; this shift was accompanied by accumulation of regulatory $\mathrm{T}$ cells (Tregs) in the spleens of infected mice and was guided by increased secretion of CC-chemokine 22 (CCL22) by splenic MФs. Importantly, neutralization of Tregs activity by co-administration of antiGITR + anti-CD25 function blocking antibodies checked the polarization of M2 M $\Phi$ to Mregs and resulted in reduced Bm-L3 burden. In conclusion, we show that Bm-L3 synergizes with host Tregs to subvert host immunity and establish itself during the first week of infection. Strategies that can prevent the polarization of host MФs at the earliest host-parasite interface can help control or limit the progression of the disease.

\section{MATERIALS AND METHODS}

\section{Animals and Parasite}

6-8 weeks old female BALB/c mice were used for all the experiments in accordance with our Institutional Animal Ethics Committee (IAEC) guidelines. Animals were housed in polypropylene cages (five animals per cage) and kept at our institute's laboratory animal facility under standard pathogen-free conditions of temperature $\left(24 \pm 1^{\circ} \mathrm{C}\right)$ and humidity (55-68\%) and fed standard pellet diet and water ad libitum. Brugia malayi was maintained in Mastomys coucha and the third infective larval stage of the parasite (Bm-L3, $n=50)$ recovered from infected Aedes aegypti were used to infect mice via the intra-peritoneal (i.p.) route. Control animals were administered sterile PBS (i.p.).

\section{Reagents}

cDNA synthesis kit, SYBR green master mix, Trizol reagent, DQ-ovalbumin, anti-mouse monoclonal antibodies viz. F4/80, toll-like receptor (TLR)-2, TLR-4, TLR-6, TLR-9, CD69, FITClabeled secondary IgG antibody, Annexin V Apoptosis Detection
Kit, and Caspase sampler assay kit were purchased from Thermo Fischer Scientific (Waltham, MA, USA). Function blocking antibodies viz. anti-CD25 and anti-GITR were purchased from either Thermo Fischer Scientific or BioXcell (West Lebanon, USA). May Grunwald-Giemsa stain was purchased from Merck and Co. (Darmstadt, Germany). CD11c and CD4 magnetic cell separation kit (MACS) were purchased from Miltenyi Biotec (BergischGladbach, Germany). Fixation and permeabilization kit, Brefeldin $\mathrm{A}$, cell strainer, $\mathrm{RBC}$ lysis buffer, anti-mouse monoclonal antibodies viz. CD11c, CD11b, CD80, CD86, MHC-II, TNF- $\alpha$, IL-4, IL-12, IL-10, Gr-1, Siglec-F, CD4, CD25, FoxP3, CD19, CD5a, and CD1d were purchased from BD Biosciences (San Jose, CA, USA). Anti-mouse monoclonal antibodies Arginase-1 and NOS2 and CCL22 ELISA kit were purchased from R\&D biosystems (Minneapolis, MN, USA). NF- $\mathrm{kB}$, p-p38, and p-ERK antibodies were purchased from Cell Signaling Technology (Danvers, MA, USA). MEK inhibitor PD0325901, protein tyrosine phosphatase (PTP) inhibitor [bpv (phen)], FITC-dextran, and Arginase activity kit were purchased from Sigma (St. Louis, MO, USA). ELISA kits for Prostaglandins E2 (PGE2) and PGD2 were purchased from Cayman chemicals (Ann Arbor, MI, USA). ELISA kits for LXA4 and LXB4 were purchased from Elabscience (Bethesda, MD, USA). Tyrosine Phosphatase Assay kit was purchased from Promega (Madison, USA).

\section{Flow Cytometry}

CD11c positive cells were enriched from the spleens of uninfected and Bm-L3 infected mice at day 3 and day 7 post infection using CD11c magnetic beads as described earlier (6). Thereafter, CD11c positive cell fraction (containing mostly DCs and MФs) was incubated with CD11b-PE-Cy7 and F4/80-Pacific Blue anti-mouse monoclonal antibodies for $20 \mathrm{~min}$ at $4^{\circ} \mathrm{C}$ and flow cytometric data were acquired on five-decade log-scale dot plots displaying forward scatter (FSC) area vs. side scatter area (SSC). First hierarchy gate was set in FSC-A vs. SSC-A dot plot to exclude contaminating dead cells and cell debris, second hierarchy gate was set in FSC-A vs. FSC-H dot plot to exclude cell doublets and, thereafter, splenic MФs present within the FSC-A vs. FSC-H dot plot were identified as $\mathrm{CD} 11 \mathrm{~b}^{\mathrm{pos}}, \mathrm{F} 4 / 80^{\text {pos }}$ cells as outlined in Figure 1A. Similarly, eosinophils, inflammatory monocytes, and regulatory B-cells (Bregs) were immunophenotyped from erythrocyte free single cell suspension of spleens based on the differential expression of following markers viz. Siglec F, MHC II, Gr-1, CD11b, CD19, CD1d, and CD5. Briefly, dot plot of FSC-A vs. SSC-A was used to identify granulocytes (low FSC-A, high SSC-A), and further sub-gating of this population helped in the identification of eosinophils (Siglec $\mathrm{F}^{\text {hi }}$, MHC $\mathrm{II}^{\text {neg }}$ ). Monocytes were identified as $\mathrm{Gr}-1^{\text {hi }}$, CD11 b pos cells, while Bregs were identified as $\mathrm{CD} 19^{+}, \mathrm{CD} 1 \mathrm{~d}^{\mathrm{mid}}, \mathrm{CD} 5 \mathrm{a}^{\text {hi }}$ cells. Data were acquired on FACS Aria flow cytometer and compensation and data analysis was done using FACS DiVA software (BD Biosciences).

\section{Flow Cytometry Assisted Sorting of Splenic MФs}

Splenic MФs were identified using the gating strategy as described above. MФs were sorted using a high-speed FACS Aria flow 
A

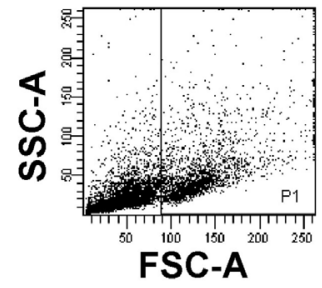

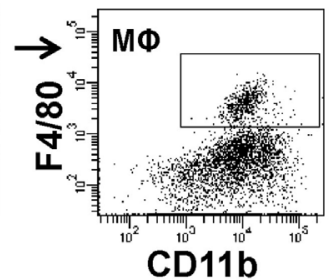

Post sort analysis

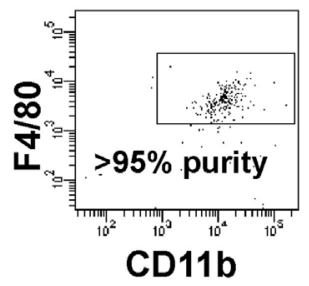

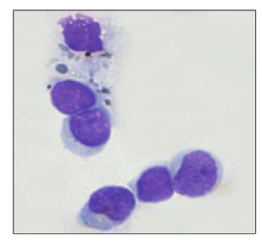

B

IL-12
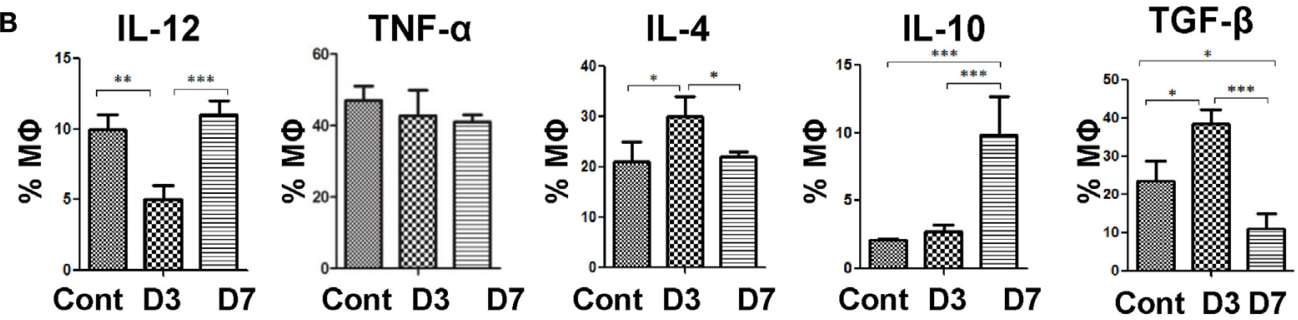

C

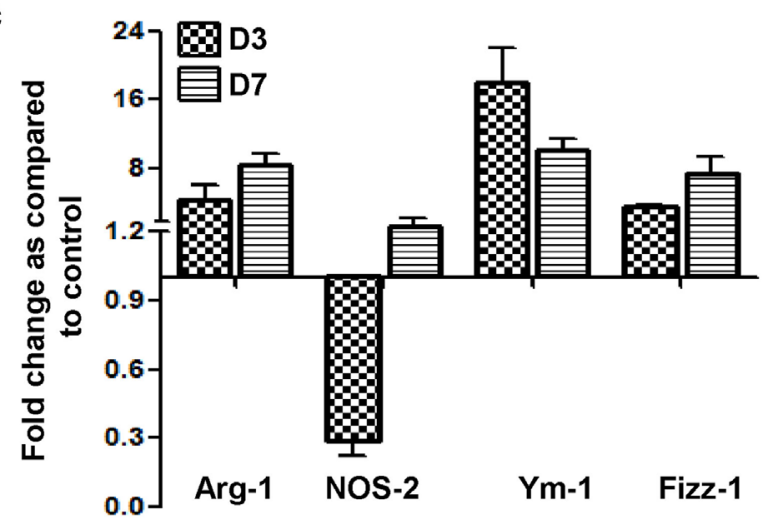

D

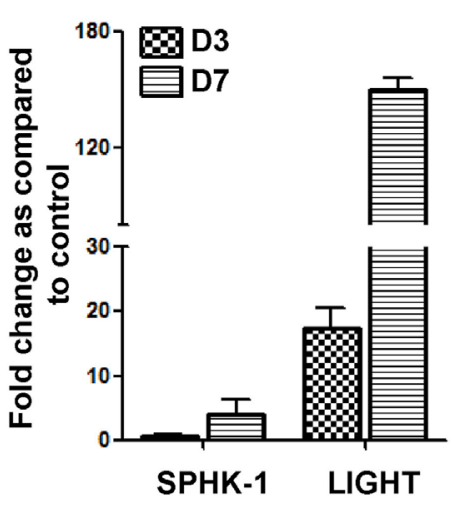

E

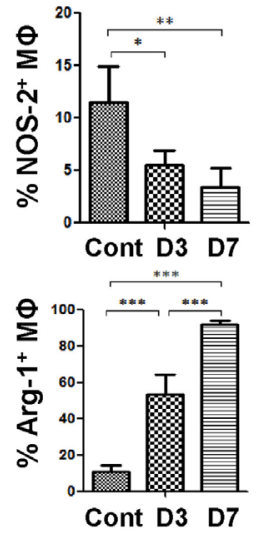

$\mathbf{F}$

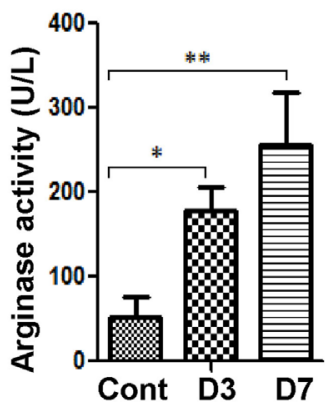

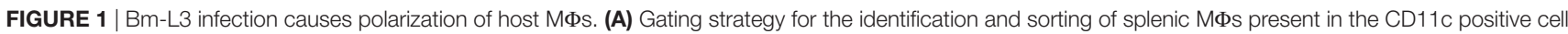
fraction is shown. Post sort dot plot, and May-Grunwald-Giemsa-stained cytospins illustrate very high purity ( $\geq 95 \%)$ of sorted cells. (B) Percentages of splenic MФs secreting IL-12, TNF- $\alpha$, IL-4, IL-10, and TGF- $\beta$ at indicated time points are shown. (C,D) Bar graphs represent fold change in the transcript levels of Arg- 1 , iNOS, Ym-1, and Fizz-1 (C) and Sphingosine kinase-1 (SPHK-1) and LIGHT (D) from FACS-sorted splenic MФs at day 3 and day 7 post Bm-L3 infection as compared to control. The value set to 1 indicates no regulation, while values $<1$ indicate respective downregulation and values $>1$ indicate respective upregulation of a given gene at indicated time points post Bm-L3 infection. (E) Representative histograms show intracellular expression of Arg-1 and NOS-2 in splenic MФs from uninfected control and Bm-L3-infected mice at day 3 and day 7 p.i. as determined by flow cytometry. Bar graphs on the right depict percentage (Mean \pm SD) values of Arg-1+ and NOS-2+ splenic MФs (F) Arginase activity in FACS-sorted splenic MФs from uninfected control and Bm-L3 infected mice at day 3 and day 7 postinfection is shown. All values represent mean \pm SD values from three independent experiments with at least $3-4$ animals/group. $p$-Value of $\leq 0.05, \leq 0.01$, and $\leq 0.001$ was considered significant, highly significant, and very highly significant and marked with ${ }^{*},{ }^{* \star}$, and ${ }^{\star \star \star}$, respectively. 
cytometer fitted with a $70 \mu \mathrm{m}$ nozzle as described recently (6-8). Sorted MФs were subjected to post-sort analysis to ascertain the purity of sorted cells and a small fraction was used to prepare cytospins. Remaining cells were used for other immunological assays as detailed below.

\section{Estimation of Toll-Like Receptors (TLRs) and Intracellular Cytokines}

CD11 $c^{\text {pos }}$ cells $\left(n=4 \times 10^{6}\right)$ were stained with CD11b-PE-Cy7 and F4/80-Pacific Blue monoclonal antibodies and distributed into four separate tubes followed by addition of FITC-labeled anti-mouse TLR-2, TLR-4, TLR-6, or TLR-9 antibodies. TLR expression on splenic M $\Phi$ s was ascertained using the gating strategy as described above (Figure 1A). For estimating intracellular cytokines, CD11 $\mathrm{c}^{\text {pos }}$ cells were first incubated with Brefeldin A $(10 \mu \mathrm{g} / \mathrm{ml})$ for $6 \mathrm{~h}$, then washed and stained with CD11b-PE-Cy7 and F4/80-Pacific blue monoclonal antibodies. Next, they were fixed, permeabilized, and stained with PE-labeled anti-mouse TNF- $\alpha$, TGF- $\beta$, IL-12, IL-4, and IL-10 monoclonal antibodies in separate tubes. Data were acquired on FACS Aria flow cytometer and compensation and data analysis was done using FACS DiVA software (BD Biosciences) $(6,7,9)$.

\section{Analysis of Co-Stimulatory Molecules, Phagocytosis, and Antigen-Processing Capacity of Splenic MФs}

FACS-sorted splenic M $\Phi$ s $(n=50,000)$ were incubated with either FITC-dextran $(1 \mathrm{mg} / \mathrm{ml})$ to estimate phagocytosis capacity, or with DQ-ovalbumin $(0.5 \mathrm{mg} / \mathrm{ml})$ to measure antigen processing capacity for $1 \mathrm{~h}$ in a $\mathrm{CO}_{2}$ incubator at $37^{\circ} \mathrm{C}$ and increase in FITC fluorescence was measured as described earlier (7). For analysis of maturation and co-stimulation markers, FACS-sorted splenic MФs were incubated with anti-mouse monoclonal antibodies viz. CD80, CD86, and $\mathrm{MHC}-\mathrm{II}$ at $4^{\circ} \mathrm{C}$ for $20 \mathrm{~min}$ and, thereafter, acquired on FACS Aria flow cytometer.

\section{T-Cell Proliferation Capacity of Splenic MФs}

FACS-sorted splenic MФs $(n=5,000)$ were cocultured with $\mathrm{CD}^{+} \mathrm{T}$ cells $\left(n=2 \times 10^{5}\right)$ that were purified from the spleens of naïve mice using CD4 magnetic beads (Miltenyi Biotec). After $48 \mathrm{~h}$, mitochondrial activity as a measure of $\mathrm{T}$ cell proliferation was measured by XTT assay (6).

\section{ELISA}

Lipid intermediates viz. PGE2, PGD2, Lipoxin A4 (LXA4), and B4 (LXB4) present in the culture supernatant of flow-sorted splenic MФs from control and Bm-L3-infected mice were quantified by ELISA as per instructions of the respective manufacturer.

\section{Inhibitor Studies}

Bm-L3-infected mice were administered either MEK inhibitor, PD0325901 (5 mg/kg, i.p.) or PTP inhibitor [bpv (phen)] (0.8 mM, i.p.) in separate experiments. In one set of experiments, Bm-L3-infected mice received PD0325901 at day 0 and were sacrificed at day 3 p.i., while in another set they received two doses of PD0325901 at day 0 and day 3 p.i. and were sacrificed at day 7 p.i. Similarly, for PTP inhibition studies, Bm-L3 infected mice received $0.8 \mathrm{mM}$ of [bpv (phen)] daily between day 4 and day 6 p.i. and were sacrificed at day 7 p.i. Bm-L3-infected mice that did not receive any inhibitor served as control animals for inhibition studies.

\section{Real-time RT PCR}

Total RNA was isolated, quantified, and reverse transcribed from FACS-sorted splenic MФs and reactions were run on Step One plus thermal cycler (Applied Biosystems) using the SYBR green master mix as described earlier (10). $\beta$-actin was used as the reference gene and mean fold-changes were calculated according to the $2^{-\Delta \Delta C T}$ method (11). After reactions were over, melting curve analysis was performed to confirm the specificity of amplicons. Primer sequences used for RT-PCR are listed in Table $\mathbf{1 .}$

TABLE 1 | Primer sequences used for real-time RT-PCR.

\begin{tabular}{|c|c|c|}
\hline Gene & Forward primer $\left(5^{\prime}-3^{\prime}\right)$ & Reverse primer $\left(5^{\prime}-3^{\prime}\right)$ \\
\hline Ptpr a & TGGTTCATTCTTGTCCTGTITGG & CTGTAGTAGCATTGTTGGCACT \\
\hline Ptpr b & TCAAGGCAGGACAGTACCC & TGTATITCTCCCATTCGCCTAGA \\
\hline Ptpr c & CAGAAACGCCTAAGCCTAGTTG & ATGCAGGATCAGGTITAGATGC \\
\hline Ptpr d & AAATGGACTGAATACCGGATCAC & GAGGACCACTTGGAACATCTTC \\
\hline Ptpr e & GATGACTGCAAGCGATTCCGA & GTTCCTTGTATGTGTCCAGATGG \\
\hline Ptpr $f$ & CTGCTCTCGTGATGCTTGGTT & ATCCACGTAATTCGAGGCTTG \\
\hline Ptpr g & AGTCAGTCCGAGGGACAATTC & GGTGGCGTAGTCAAGGAGC \\
\hline Ptpr h & СTTCTGGGGGATATGGGGTC & TCCCACACAAAGATCCAППTCA \\
\hline Ptpr j & GACTCAGGCGCTTCAGAATGT & TTGTTCAAGGTCTCATTGGTTGT \\
\hline Ptpr k & СTCTGGCTTCTGTACCCGTG & GGACCCACTCAAAGTCATCGTAT \\
\hline Ptpr m & CCGGAGAAACATITCAGGTGG & AGGTCGGTITGGTCAGGGT \\
\hline Ptpr $n$ & CTTGTGTATGCGCCATCATTCG & GGTCCTGGTACTCAAAAGTAGTG \\
\hline Ptpr n2 & GAGGATGGCTTGTGTGGATCA & CGGAACCTITGACATCTTCCAA \\
\hline Ptpr o & GCACACTITAATTGGACTGCTC & TGCCAGCTCCACATTCCCTA \\
\hline Ptpr $r$ & GAACGTGGTTGTGGACCCTC & TGGAGTCCTATGGGCTTCATT \\
\hline Ptpr s & GGTGAACAACATACCCCCGAC & TCCCACСTCTGTGTAAGCCA \\
\hline Ptpr t & CCATGCAGAAAAGCACCTCAT & GGGACCACTTTCCA \\
\hline Ptpr u & GGCTCAGTATGACGACTTCCA & GAACTGCACACAATGGGTGTC \\
\hline Ptpr z1 & GGAGAAGAACAGAACATCGTCC & TCATTGCTCTGGTAATAGCCCA \\
\hline Ptp n1 & GGAACTGGGCGGCTATTTACC & CAAAAGGGCTGACATCTCGGT \\
\hline Ptp n11 & ATGTGTCA & CTGTGTTCCTTGTC \\
\hline Ptp n12 & ATGGAGCAAGTGGAGATCCTG & TCTCAATCGCATGAAGTCCCG \\
\hline Ptp n13 & SAGAGTGC & CGGTGCGGT \\
\hline Ptp n14 & ACAGAAGAGACCGCTGATGTT & CTCTGAGCCTCTCGTCTATGG \\
\hline Ptp n2 & GCAGTGAGAGCATTCTACGGA & TGACACAAACCCCATCTTAGTGA \\
\hline Ptp n21 & TTGAAATTGAAACGCACCCGA & GCCACAGCTTCTAGGCTCTC \\
\hline Ptp n22 & CAGCAACTACTGAAAGAAGCCC & AGGATAGATITTGTCGGCCTTG \\
\hline Ptp n23 & ACAGAATGCTATTCGCGTTGC & СCTGCTCGTACTTGATGTCTTC \\
\hline Ptp n3 & TTACGTGCGTTGGGTGGAAG & AGCCATCTAAAAACCGTATGCTG \\
\hline Ptp n4 & TCCGCCTGGATAGACCACTT & GCCATATTGAACTGACTGGACTT \\
\hline Ptp n5 & TACCAAGTCTGCTGCTAGTCT & GCTCACTGATTGACGCCTGT \\
\hline Ptp n6 & GGACTTCTATGACCTGTACGGA & GCTGCGTGTAATACTCGACCA \\
\hline Ptp n7 & CTGCCGACCTTGTCTITGG & AGCAGATGGGTTCAACCGTG \\
\hline Ptp n9 & TTCCACTGCTACAGAGAAACAAG & GTTGGGTCTCGAACATTCAAGAT \\
\hline Ptp 4a1 & CAATCCAACCAATGCGACCTT & GAGTAGTGTCGTAAGTTGCTTCA \\
\hline Ptp $4 a 2$ & CCACCAATGCGACTCTCAACA & CCATCATCAAACGGCCAATCT \\
\hline Ptp $4 a 3$ & CATCACTGTTGTGGACTGGC & TGGATGGCGTCCTCGTACTT \\
\hline Ptp la & GCCAGCGACGAGAAGGAAG & CCGTCATGGCGATATTGTAGAA \\
\hline Gapdh & AGGTCGGTGTGAACGGATTTG & TGTAGACCATGTAGTTGAGGTCA \\
\hline Arg-1 & CTCCAAGCCAAAGTCCTTAGAG & AGGAGCTGTCATTAGGGACATC \\
\hline iNOS & GGAGTGACGGCAAACATGACT & TCGATGCACAACTGGGTGAAC \\
\hline$Y m-1$ & CAGGTCTGGCAATTCTTCTGAA & GTCTTGCTCATGTGTGTAAGTGA \\
\hline Fizz-1 & CCAATCCAGCTAACTATCCCTCC & ACCCAGTAGCAGTCATCCCA \\
\hline $\begin{array}{l}\text { Sphingosine } \\
\text { kinase-1 }\end{array}$ & TCCTGGAGGAGGCAGAGATA & GCTACACAGGGGTTCTGGA \\
\hline LIGHT & CTGCATCAACGTCTTGGAGA & GATACGTCAAGCCCCTCAAG \\
\hline
\end{tabular}




\section{Analysis of Intracellular Cell Signaling Molecules}

FACS-sorted splenic MФs $(n=200,000)$ were fixed, permeabilized, and stained with primary antibodies against p-p38 and p-ERK. Thereafter, FITC-labeled secondary IgG antibody was added, and after a brief incubation, cells were washed and acquired on a BD FACS Aria flow cytometer (12). For analysis of transcription factor NF- $\kappa B$, flow-sorted splenic $М \Phi s$ were lysed in RIPA buffer containing protease inhibitor cocktail and resolved on 10\% SDS-PAGE followed by transfer onto nitrocellulose membrane. Next, membranes were blocked with $3 \%$ BSA and probed overnight with primary antibody followed by addition of HRP-conjugated secondary antibody and detected using ECL kit.

\section{Enzymatic Assays}

FACS-sorted splenic MФs $\left(n=2 \times 10^{6}\right)$ were lyzed in RIPA buffer containing protease inhibitor cocktail and centrifuged at $10,000 \times g$ for $15 \mathrm{~min}$ at $4^{\circ} \mathrm{C}$. Protein tyrosine phosphatase (PTP) activity was determined in the supernatant according to instructions of the manufacturer (13). Arginase activity was similarly measured in the supernatant by comparing with a urea standard (14). For quantification of caspase activity, p-nitroaniline labeled peptide substrate was used and absorbance of free pNA was quantified using a spectrophotometer (15).

\section{Neutralization of Regulatory T Cells}

To neutralize the activity of regulatory $\mathrm{T}$ cells, Bm-L3-infected mice were administered either respective IgG isotypes or combination of anti-CD25 + anti-GITR function blocking antibodies (100 $\mu$ g each, i.v.) at day 4 and day 6 post Bm-L3 infection, $24 \mathrm{~h}$ later, spleens were excised and immunological assessments were carried out as described in the study.

\section{Apoptosis of Regulatory T Cells}

Apoptosis of Tregs was assessed by Annexin V-FITC and propidium iodide (PI) staining pattern of $\mathrm{CD} 4{ }^{+} \mathrm{CD} 25^{+} \mathrm{T}$ cells (Tregs) present within the lymphocyte gate (low FSC-A, low SSC-A) as per the instructions of the manufacturer.

\section{Statistics}

Figures represent mean \pm SD values derived from at least three independent experiments having at least 3-4 animals/group. Statistical analysis was done by either one-way analysis of variance using Bonferroni's test (Graph Pad Prism 5.0 version 5.01; Graph Pad, San Diego, CA, USA) or two tailed Student's $t$-test. $p$-Value $\leq 0.05, \leq 0.01$, and $\leq 0.001$ between different groups or time points were considered significant, highly significant and very highly significant and marked with ${ }^{\star},{ }^{* *}$ and ${ }^{* * *}$ respectively.

\section{RESULTS}

\section{Infective Larvae of Brugia malayi (Bm-L3) Polarize Splenic MФs}

Splenic $М \Phi$ s were immunophenotyped and sorted using flowcytometry on day 3 and day 7 post Bm-L3 infection as described in the Section "Materials and Methods." Post-sort analysis showed very high purity (>95\%) of sorted cells (Figure 1A). Percentages of splenic MФs-secreting IL-4 increased at day 3 p.i. $(p \leq 0.05)$, but those secreting IL-12 decreased $(p \leq 0.01)$ as compared to uninfected controls. This scenario changed rapidly at day 7 p.i. when splenic MФs secreting IL-4 decreased significantly ( $p \leq 0.05)$ while those secreting IL-12 and IL-10 increased as compared to day 3 p.i. ( $p \leq 0.001$ for both). Notably, percentages of splenic MФs secreting TGF- $\beta$ increased at day 3 p.i. $(p \leq 0.05)$ but dropped significantly at day 7 p.i. ( $p \leq 0.001$, Figure 1B).

Real-time RT-PCR analysis revealed 4.5-fold elevated mRNA expression of Arg-1 at day 3 p.i., which increased further to 8.5-fold at day 7 p.i. Quite contrary to this, mRNA expression of NOS2 in splenic MФs decreased 3.5-fold at day 3 p.i., but moderately increased to 1.8 -fold at day 7 p.i. when compared to uninfected controls (Figure 1C). Importantly, transcript levels of other alternative activation markers viz., Ym-1, which is associated with eosinophil chemotaxis increased 18 -fold at day 3 p.i. and 10 -fold at day 7 p.i. Similarly, Fizz-1, which dampens Th2 inflammation was upregulated 3.6- and 7.5-fold at day 3 and day 7 p.i., respectively (Figure 1C). Also, key markers asociated with regulatory macrophages, i.e., sphingosine kinase-1 and LIGHT were upregulated by 4 - and 150 -fold, respectively, at day 7 p.i. (Figure 1D), suggesting that Bm-L3-infected splenic MФs were indeed phenotypically different at these two time points.

To further confirm our findings, we analyzed expression levels of Arg-1 and NOS2 via flow cytometry and the results showed 4.7and 8-fold upregulated levels of Arg-1 in Bm-L3-infected splenic MФs at day 3 and day 7 p.i., respectively, which was in contrast to 2.0- and 3.4-fold downregulated levels of NOS2 observed at day 3 and day 7 p.i., respectively (Figure 1E). Interestingly, this observation was corroborated by 3.5 -fold $(p \leq 0.05)$ and five fold $(p \leq 0.01)$ increased arginase activity in Bm-L3-infected splenic MФs at day 3 and day 7 p.i., respectively, as compared to uninfected control animals (Figure 1F). Taken together, these results reflected early perturbations in the cytokine secreting potential of splenic MФs and underlined a tactical shift from a predominantly alternatively activated state (M2 phenotype) of splenic MФs at day 3 p.i. to a more polarized regulatory phenotype (Mregs) at day 7 p.i. during the first week of Bm-L3 infection.

\section{Polarized Splenic MФs Are Differentially Impaired during Bm-L3 Infection}

We assessed the expression of maturation and co-stimulatory markers on the two phenotypes of splenic MФs, i.e., M2 MФs and Mregs at day 3 and day 7 p.i., respectively. While the expression of MHCII and CD86 decreased in M2 MФs, that of CD80 did not change significantly at day 3 p.i. However, expression of these markers was elevated in Mregs at day 7 p.i. (Figure 2A). Furthermore, M2 MФs exhibited decreased phagocytic capacity (Figure 2B) ( $p \leq 0.05$ ), which contrasted with significantly increased antigen processing capacity $(p \leq 0.001)$ observed in Mregs at day 7 p.i. (Figure 2C).

To further understand the functional consequences of these findings on the proliferation of T cells, FACS-sorted M2 MФs (at day 3 p.i.) and Mregs (at day 7 p.i.) were cocultured with purified $\mathrm{CD} 4^{+} \mathrm{T}$-cells obtained from naïve mice. Results showed that 

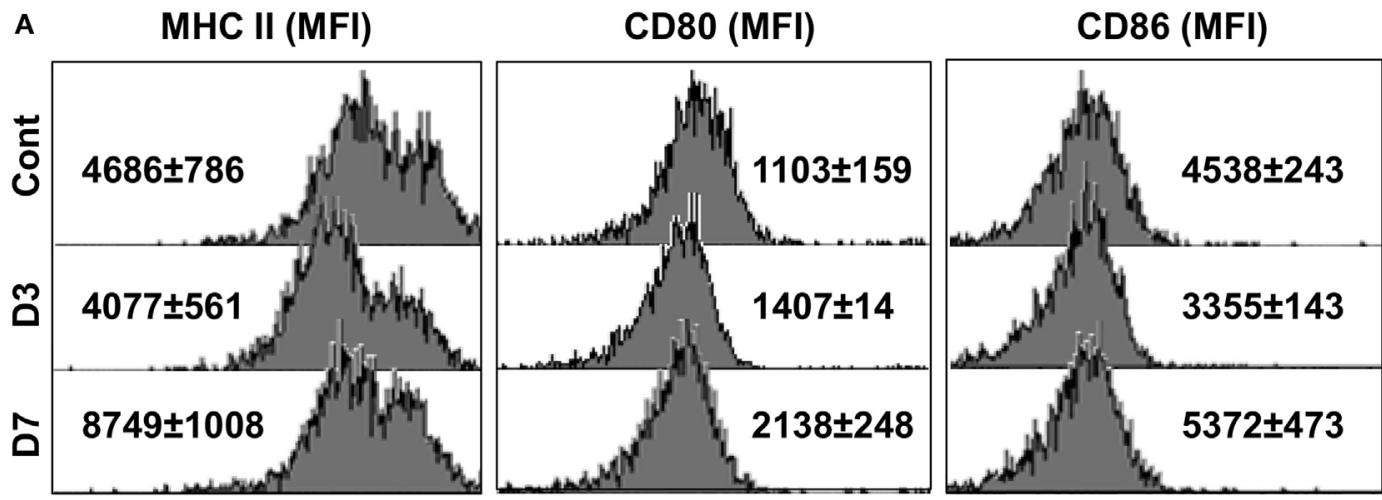

B

C

D
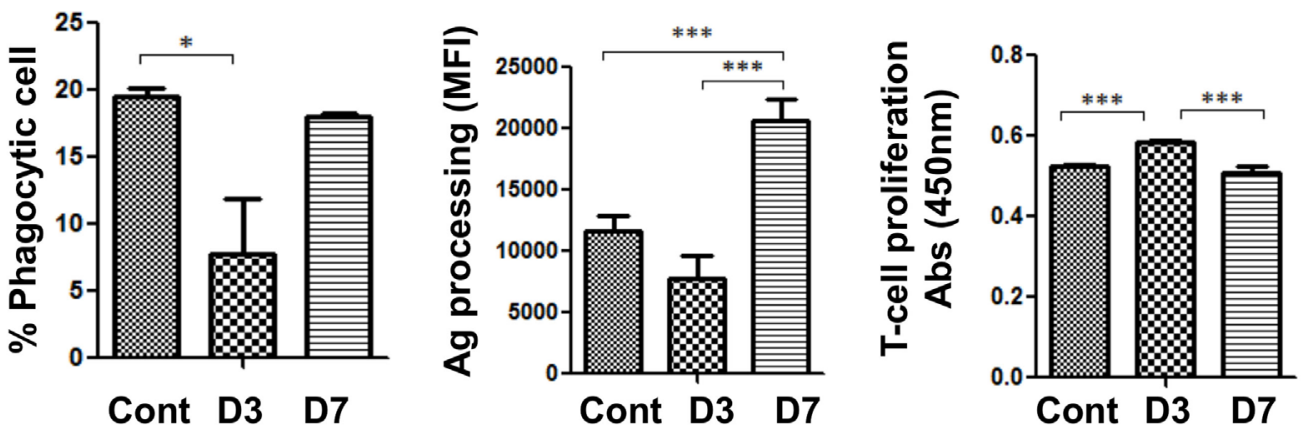

E
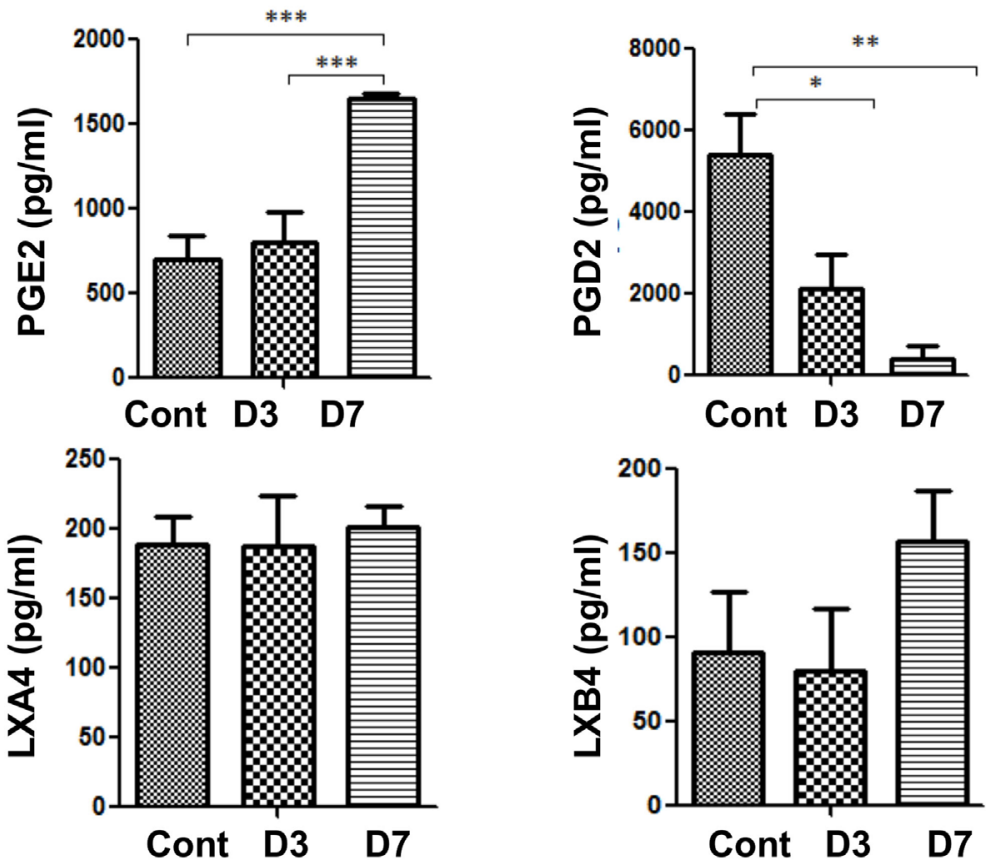

FIGURE 2 | Bm-L3 infection differentially modulates the functions of host MФs. (A) Histograms represent expression of maturation and costimulatory markers viz. MHC II, CD80, and CD86 on FACS-sorted splenic MФs from uninfected control and Bm-L3 infected mice at day 3 and day 7 p.i. as determined by flow cytometry. Values in bracket are mean fluorescence intensity (MFI) values at given time points. (B,C) Antigen uptake (phagocytosis) and antigen-processing capacity of FACS-sorted splenic MФs from uninfected control and Bm-L3-infected mice at day 3 and day 7 postinfection is shown. Values represent \% phagocytic cell or MFI values as determined by FITC-Dextran uptake or DQ-ova-albumin cleavage, respectively. (D) Mitochondrial activity, as a measure of T cell proliferation was assessed

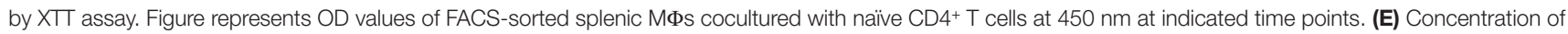
different lipid intermediates present in the culture supernatant of FACS-sorted splenic MФs is shown. All values represent mean \pm SD values from three independent experiments with at least $3-4$ animals/group. $p$-value of $\leq 0.05, \leq 0.01$, and $\leq 0.001$ was considered significant, highly significant, and very highly significant and marked with *, ${ }^{* *}$, and ${ }^{* \star}$, respectively. 
M2 MФs supported the proliferation of $\mathrm{CD} 4^{+} \mathrm{T}$ cells (Figure 2D; $p \leq 0.001)$ Mregs did not, which underlined the suppressive nature of these cells. Moreover, significantly elevated level of Prostaglandin E2 (PGE2, $p \leq 0.001$ ), but drastically decreased level of Prostaglandin D2 (PGD2, $p \leq 0.01$ ) was observed in Mregs (Figure 2E), but quite surprisingly, no significant change was observed in the level of other small lipid intermediate viz. Lipoxin A4 (LXA4), and Lipoxin B4 (LXB4). These results showed that in addition to IL-10, elevated levels of PGE2 might have contributed toward polarization of M2 MФs to Mregs during the early phase of Bm-L3 infection.

\section{MAP Kinases Regulate Polarization of Splenic MФs}

To gain deeper insights into the molecular pathways that regulated the polarization of splenic $M \Phi$ s, we assessed the expression of toll-like receptors on MФs via flow cytometry. Results presented as histograms (Figure 3A) along with mean fluorescence intensity (MFI) values given in Table 2 showed that expression of TLR2, TLR4, TLR6, and TLR9 increased in M2 MФs and Mregs at day 3 at day 7 p.i. as compared to uninfected controls. However, no change was observed in the transcript level of TLR 5 between M2 MФs and Mregs (data not shown). Furthermore, while the expression of p-p38 remained unaltered, that of p-ERK was significantly upregulated in M2 MФs at day 3 p.i. (Figure 3B; $p \leq 0.001$; MFI = 6,139 \pm 785 ); however, it was downregulated in Mregs at day 7 p.i. (Figure 3B; $p \leq 0.01$, MFI $=3,611 \pm 164)$ when compared to uninfected controls (Figure 3B; MFI = 2,885 \pm 243 ).

Additionally, since the activation of MAPKs is counterregulated by several PTPs, we estimated PTP activity and found that it was significantly upregulated in Mregs at day 7 p.i. as compared to M2 МФs (Figure 3C, $p \leq 0.01$ ). Further mRNA analysis revealed that six different PTPs viz., Ptprh, Ptprn, Ptprr, Ptpn21, Ptpn23, and Ptp4a were significantly upregulated in Mregs, which suggested their involvement in inducing hypo-responsive state in Mregs (Figure 3D).

We also analyzed the phosphorylation pattern of two major subunits of NF- $\mathrm{kB}$ viz. p65 and p50/105 and found that expression of p65 and p50/105 was downregulated in M2 MФs, but it was severely impaired in Mregs (Figure 3E). To confirm whether MAPKs regulated this process of polarization, we administered MEK inhibitor PD0325901 to Bm-L3-infected mice and found that ERK inhibition did not affect the development of M2 phenotype, but it significantly altered the polarization of M2 MФs to Mregs as was evident from higher percentages of IL-4-secreting MФs $(p \leq 0.05)$ at D7 post PD0325901 treatment (Figure 3F). Interestingly, administration of PD0325901 did not affect the IL-10 secreting potential of MФs following treatment. Similarly, to confirm the role of PTPs in the polarization of M2 MФs to Mregs, we administered PTP inhibitor [bpv (phen)] to Bm-L3-infected mice daily between day 4 and day 6 p.i. and found increased percentages of MФs-secreting IL-4, but not IL-10 at day 7 p.i. (Figure 3G, $p \leq 0.001$ ). These data suggest that complex mechanisms regulate the process of $M \Phi$ polarization and the parasite cleverly exploits these intricately wired molecular pathways to aid its survival.

\section{Infection with Bm-L3 Expands Regulatory T Cells in the Spleens of Mice}

Based on our previous observation of increased IL-10 secretion by Mregs at day 7 p.i., we reasoned if this would have a bearing on the numbers of Tregs present in the spleens of mice. Indeed, we observed increased percentages of Tregs at day 7 p.i. $(4.2 \pm 0.91 \%$; Figure $\mathbf{4 A})$ as compared to control animals $(2.7 \pm 0.14 \%$, Figure $4 \mathrm{~A})$. This observation was also complemented by significantly increased concentration of CCL22, a Treg chemoattractant in the culture supernatant of FACS-sorted MФs at day 7 p.i. ( $p \leq 0.01$, Figure $4 B$ ) and in the peritoneal lavage of Bm-L3-infected mice at day 7 p.i. $(p \leq 0.01$, Figure $4 \mathrm{~B})$. Together, these results supported the notion that CCL22 driven Treg recruitment might have helped in the polarization M2 MФs to Mregs. To further confirm our findings that Tregs were indeed driven by elevated CCL22 gradient and were not a result of de novo recruitment from the peripheral blood as might be the case during apoptosis, we checked the percentages of apoptotic T cells present within the $\mathrm{CD} 4{ }^{+} \mathrm{CD} 25^{+}$and $\mathrm{CD} 4{ }^{+} \mathrm{CD} 25^{-}$gate and quite expectedly found no difference between the control and Bm-L3-infected animals either at day 3 or day 7 p.i. (Figure 4C). To strengthen our reasoning even further, we blocked the activity of Tregs by co-administering neutralizing antibodies against CD25 and GITR (i.v.) at day 4 and day 6 post Bm-L3 infection (Figure 4D) and quite notably found significantly depleted percentages of Tregs (Figure 4E), along with drastically reduced Bm-L3 burden in treated animals (Figure 4F). Not only this, Bm-L3 recovered from anti-CD25 + anti-GITR treated animals were dead and surrounded by thousands of host cells attached to their surface, which showed that anti-CD25 + anti-GITR treatment suppressed the activity of Tregs and boosted anti-parasitic Th2 immunity (Figure 4G).

\section{Treatment with Anti-CD25 + Anti-GITR Antibodies Prevent Polarization of MФs}

To evaluate the effect of anti-CD25 + anti-GITR treatment on the polarization of splenic MФs, we measured IL-4 and IL-10 levels at day 7 p.i., i.e., $24 \mathrm{~h}$ after the last dose of anti-CD25 + anti-GITR antibodies and found significantly reduced IL-10 $(p \leq 0.05)$, but heightened IL-4 secretion $(p \leq 0.05)$ as compared to mice that received matching IgG isotypes (Figure 5A). Moreover, anti-CD25 + anti-GITR treatment also restored the $\mathrm{T}$ cell proliferation capacity of splenic MФs ( $p \leq 0.05$, Figure 5B) and increased the expression of T cell activation marker CD69 on $\mathrm{CD}^{+}{ }^{+} \mathrm{T}$ cells (Figure 5C). Activated $\mathrm{CD}^{+} \mathrm{T}$ cells also secreted significantly lower amount of IL-10 ( $p \leq 0.05)$ but more IL-4 $(p \leq 0.05)$ as compared to IgG controls (Figure 5D). These results further strengthened the notion that anti-CD25 + anti-GITR treatment checked the polarization of M2 MФs to Mregs and strengthened the antiparasitic immune response in treated animals.

\section{Anti-CD25 + Anti-GITR Treatment Affects the Recruitment of Eosinophil and Bregs in the Spleens of Mice}

To elucidate whether anti-CD25 + anti-GITR treatment had any effect on the accumulation of leukocytes, we carried out flow cytometry assisted multicolor immunophenotyping of different leukocyte populations present in the spleens of mice. We found 

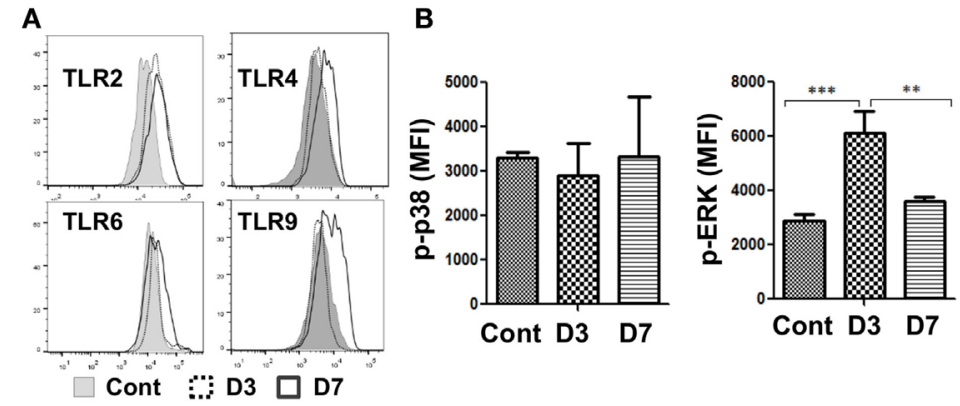

。

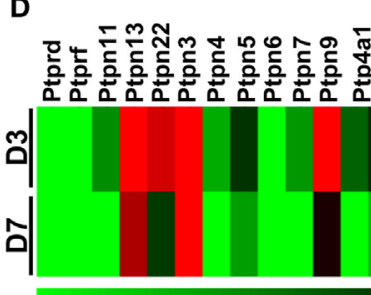

$-13.5$
B

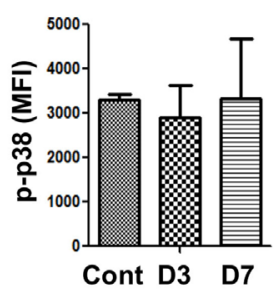

C

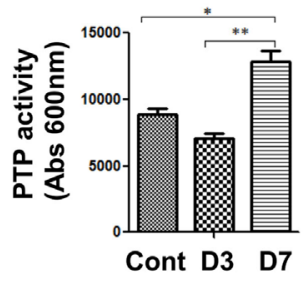

F p-ERK inhibition post Bm-L3 infection
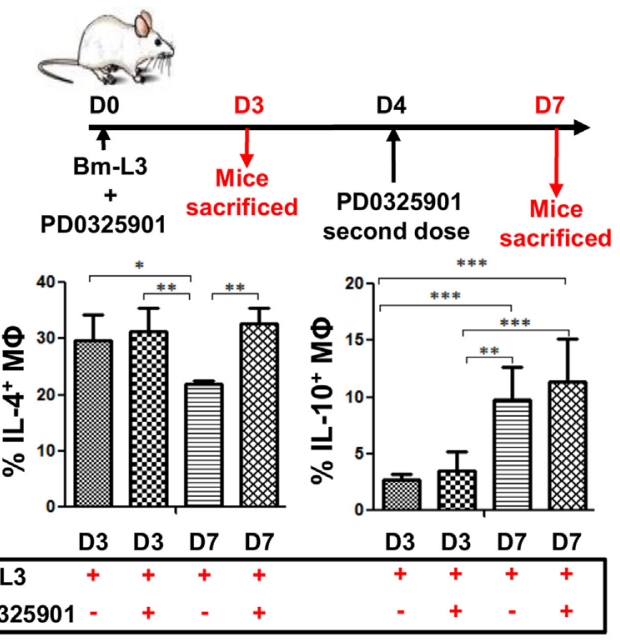

G PTP inhibition post Bm-L3 infection
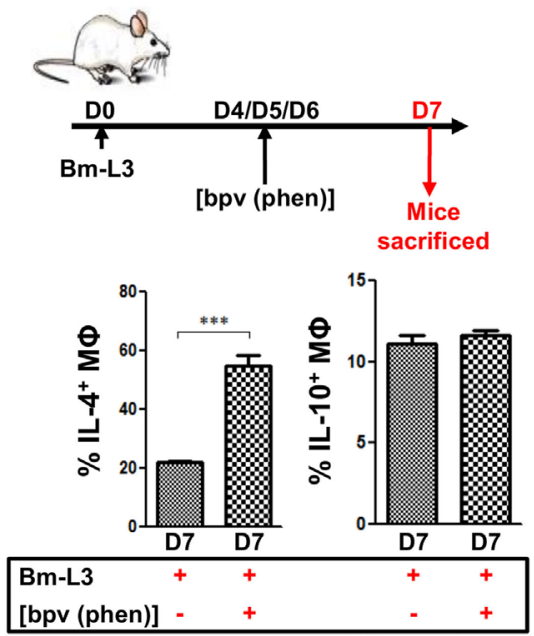

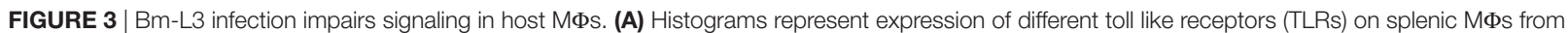
uninfected control and Bm-L3-infected mice at day 3 and day 7 p.i. (B) Phosphorylation of p38 and ERK in FACS-sorted splenic M $\Phi$ s from uninfected control and Bm-L3 infected mice at day 3 and day 7 p.i. is shown. Values represent mean fluorescence intensity (MFI) values at given time points. (C) Bar graph represents protein tyrosine phosphatases (PTP) activity in FACS-sorted splenic MФs in uninfected control and Bm-L3-infected mice at day 3 and day 7 p.i. (D) Heat map showing fold change (red-upregulated and green-downregulated of different PTPs in flow-sorted splenic MФs at day 3 and day 7 post Bm-L3 infection as compared to uninfected control). (E) Representative western blot showing expression of $\beta$-actin, NFKB-p65, and NFkB-p50/105 in uninfected control and Bm-L3-infected mice at day 3 and day 7 p.i. (F) Schematic representation of the experimental strategy used for the inhibition of Erk. Bm-L3-infected mice were administered MEK inhibitor, PD0325901 at day 0 and day 4 p.i., and immunological assessments were made at day 3 and day 7 p.i., respectively. Figure depicts the percentages of IL-4 and IL-10 secreting M $\Phi$ s at day 3 and day 7 before and after Erk inhibition. (G) Schematic representation of the experimental strategy used for the depletion of PTP activity. Bm-L3-infected mice were administered [bpv (phen)] daily from day 4 p.i. to day 6 p.i. and immunological assessments were made at day 7 p.i. Figure depicts the percentages of IL-4 and IL-10 secreting MФs at day 7 before and after PTP inhibition. Values in (B,C,F,G) represent mean \pm SD values from two independent experiments with at least $3-4$ animals/group. $p$-Value of $\leq 0.05, \leq 0.01$, and $\leq 0.001$ was considered significant, highly significant, and very highly significant and marked with ${ }^{\star},{ }^{\star \star}$, and ${ }^{* \star \star}$, respectively.

TABLE 2 | Mean fluorescence intensity values of different toll-like receptors (TLRs) in splenic MФs of control and Bm-L3 infected mice at day 3 and day 7 postinfection.

\begin{tabular}{|c|c|c|c|c|c|}
\hline & & TLR 2 & TLR4 & TLR6 & TLR9 \\
\hline Splenic MФs & Cont & $13,606 \pm 1,676$ & $3,691 \pm 141$ & $28,233 \pm 94$ & $4,945 \pm 308$ \\
\hline Splenic MФs (M2 MФs) & D3 & $18,428 \pm 1,624$ & $4,144 \pm 424$ & $31,336 \pm 4,850$ & $5,863 \pm 692$ \\
\hline Splenic MФs (Mregs) & D7 & $18,093 \pm 1,736$ & $4,384 \pm 228$ & $30,827 \pm 2,481$ & $6,095 \pm 467$ \\
\hline
\end{tabular}


A

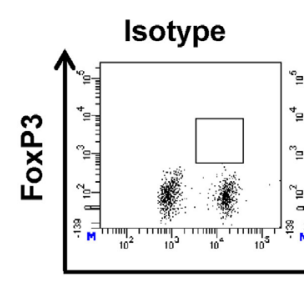

Cont

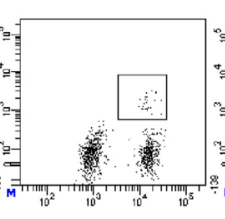

D3

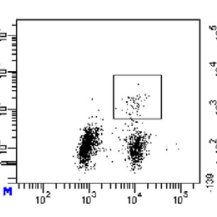

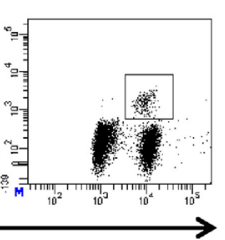

CD4

B

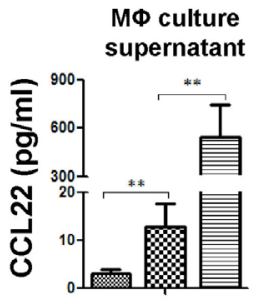

Cont D3 D7

C
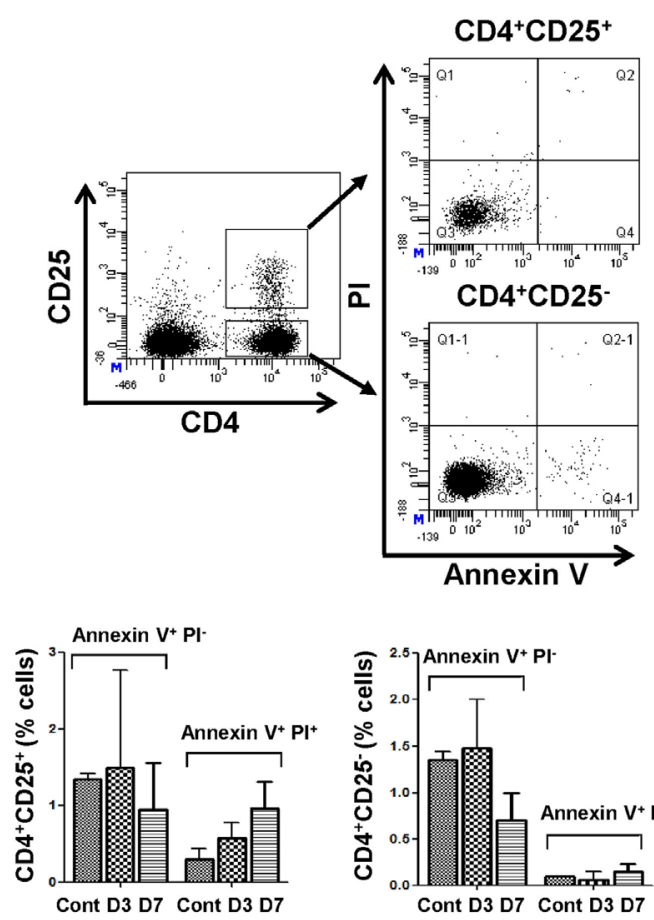

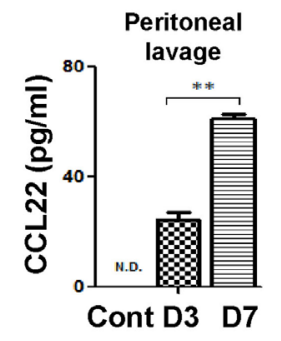

Cont D3 D7

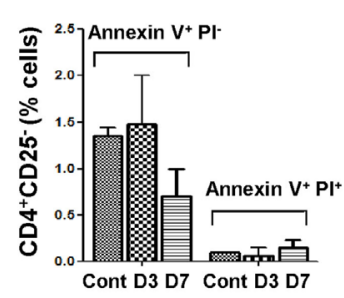

D

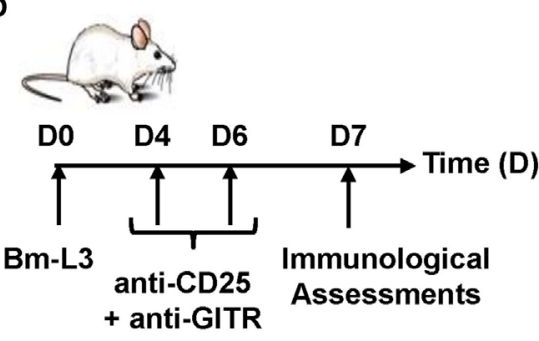

E

$\mathrm{Bm}-\mathrm{L3}$

+ anti-CD25

+ anti-GITR

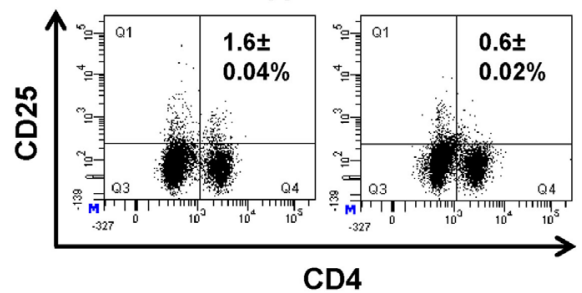

$\mathbf{F}$

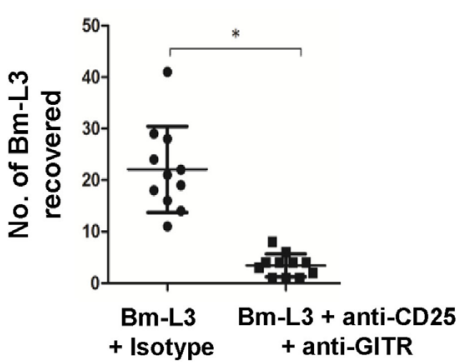

G

Bm-L3 + anti-CD25 + anti-GITR
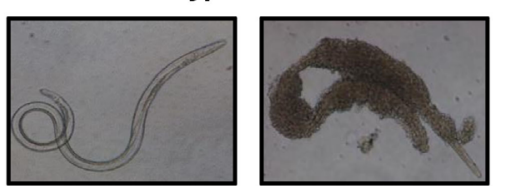

FIGURE 4 | Neutralization of regulatory T-cells (Tregs) repolarizes splenic MФs. (A) Representative flow cytometry dot plots showing CD4+Foxp3+ Tregs present in the spleens of uninfected control and Bm-L3 infected mice at day 3 and day 7 p.i. Bar graph on the right depict percentage (mean \pm SD values) of CD4+Foxp3 ${ }^{+}$Tregs. (B) Concentration of CCL22 present in the culture supernatant of FACS-sorted splenic MФs and peritoneal fluid of uninfected control and Bm-L3-infected mice at day 3 and day 7 p.i. (C) Representative flow cytometry dot plots showing apoptotic cells present within the CD4+ CD25+ and CD4+ CD25- T cell gate. Histograms show the percentages of Annexin $\mathrm{V}^{+} \mathrm{PI}^{-}$and Annexin $\mathrm{V}^{+} \mathrm{Pl}^{+}$cells present in control and Bm-L3-infected mice at day 3 and day 7 p.i., as enumerated from FACS dot plots (D) Schematic representation of experimental strategy used for the depletion of Tregs is shown. Bm-L3-infected mice were administered anti-CD25 + anti-GITR function blocking antibodies at day 4 and day 6 p.i., and immunological assessments were made at day 7 p.i. (E) Representative flow cytometry dot plots showing CD25+ CD4+ Tregs present in the spleens of anti-CD25 + anti-GITR treated mice or lgG administered controls at day 7 p.i. (F) Number of Bm-L3 recovered from the peritoneal cavity of anti-CD25 + anti-GITR-treated mice or lgG administered controls at day 7 p.i. (G) Representative bright field microscopy images showing adhesion of leukocytes (mainly eosinophils and MФs) to the dead Bm-L3 recovered from the peritoneal cavity of anti-CD25 + anti-GITR treated mice or lgG administered controls at day 7 p.i. $p$-Value of $\leq 0.05, \leq 0.01$, and $\leq 0.001$ was considered significant, highly significant, and very highly significant and marked with *, ${ }^{\star \star}$, and ${ }^{\star \star \star}$, respectively. 

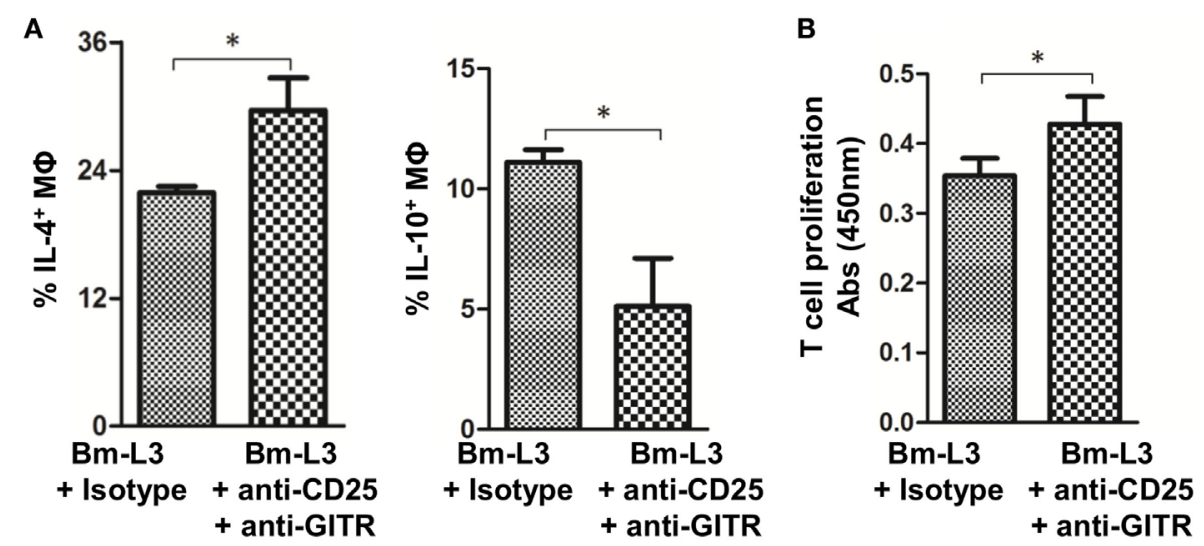

C

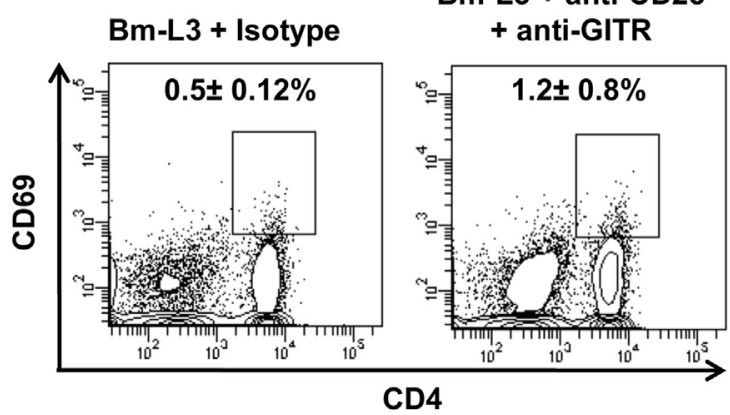

Bm-L3 + anti-CD25

+ anti-GITR
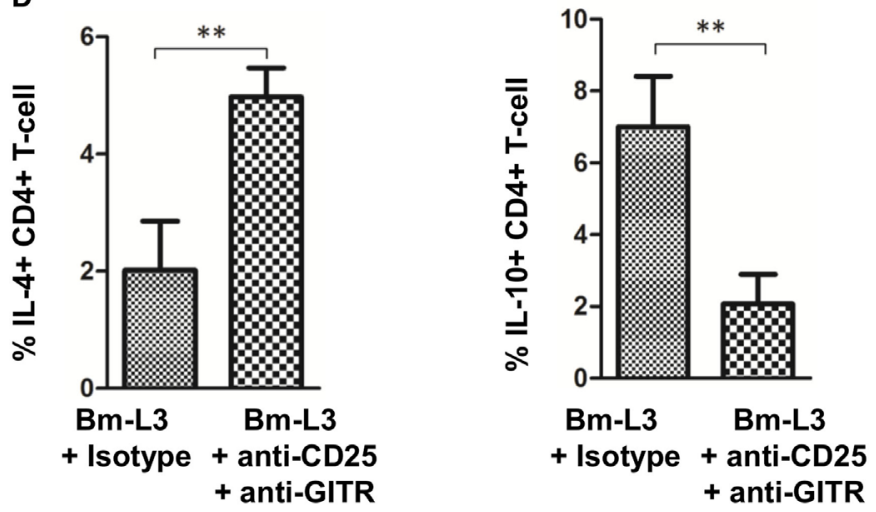

FIGURE 5 | Neutralization of regulatory T-cell activity prevents polarization of splenic MФs. (A) Bar graphs represent percentages of FACS-sorted splenic MФs-secreting cytokines IL-10 and IL-4 from anti-CD25 + anti-GITR-treated mice or uninfected IgG control at day 7 p.i. (B) Mitochondrial activity, as a measure of T cell proliferation was assessed by XTT assay. Figure represents OD values of FACS-sorted splenic MФs cocultured with naïve CD4+T cells at $450 \mathrm{~nm}$ at indicated time points from anti-CD25 + anti-GITR treated mice or uninfected lgG control at day 7 p.i. (C) Representative flow cytometry dot plots showing percentages of activated CD4+ $T$ cells from anti-CD25 + anti-GITR treated mice or uninfected lgG control at day 7 p.i. (D) Bar graphs represent percentages of CD4+ $T$ cells secreting cytokines IL-10 and IL-4 from anti-CD25 + anti-GITR treated mice or uninfected IgG control at day 7 p.i. All values represent mean \pm SD values from three independent experiments with at least $3-4$ animals/group. $p$-Value of $\leq 0.05, \leq 0.01$, and $\leq 0.001$ was considered significant, highly significant and very highly significant and marked with ${ }^{*},{ }^{* *}$, and ${ }^{* * *}$, respectively.

increased percentages of granulocytes (low FSC-A, high SSC-A) in the anti-CD25 + anti-GITR-treated mice as compared to IgG controls (Figure 6A). Further confirmation revealed that most of these granulocytes were indeed eosinophils (Figure 6B, SigF', $\mathrm{MHCII}^{-}$cells) that were present almost four times more in antiCD25 + anti-GITR-treated mice. However, unlike eosinophils, percentages of regulatory B cells $\left(\mathrm{CD} 1 \mathrm{~d}^{+}, \mathrm{CD} 5 \mathrm{a}^{\text {hi }}\right)$ and inflammatory monocytes $\left(\mathrm{Grl}^{+}, \mathrm{CD}_{11 b^{+}}\right)$decreased in anti-CD25 + anti-GITR treated mice as compared to IgG controls (Figures 6C,D). These results underlined the efficacy of anti-CD25 + anti-GITR treatment in breaking immunological tolerance in mice.

\section{In Vivo Neutralization of Tregs Delays Apoptosis of M2 MФs}

Since administration of anti-CD25 + anti-GITR antibodies helped in retaining the M2 phenotype in MФs, which 


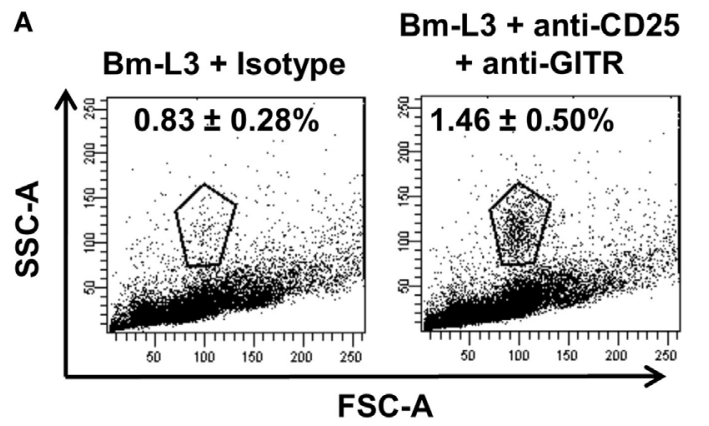

C

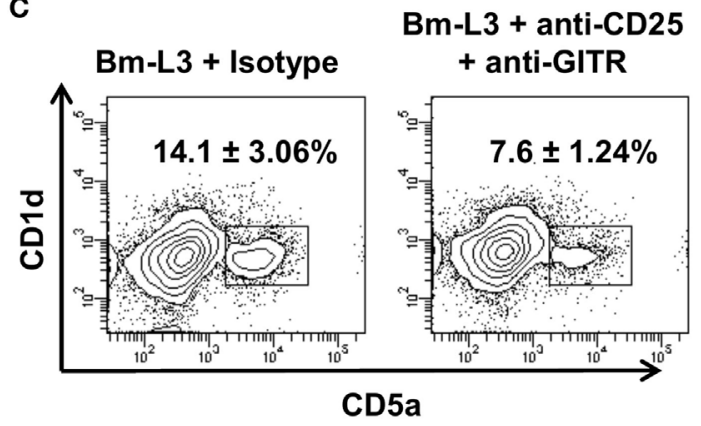

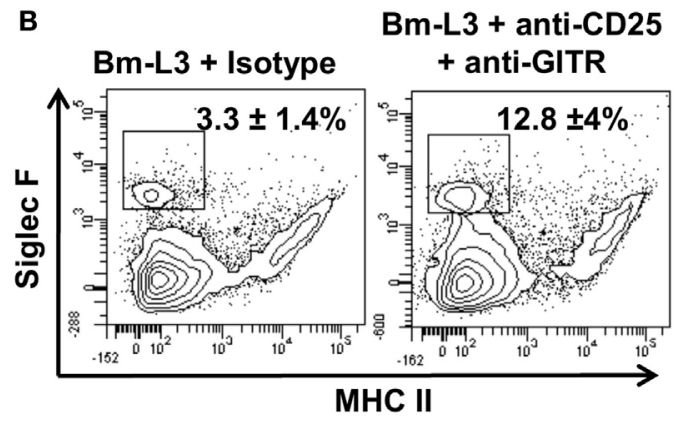

D

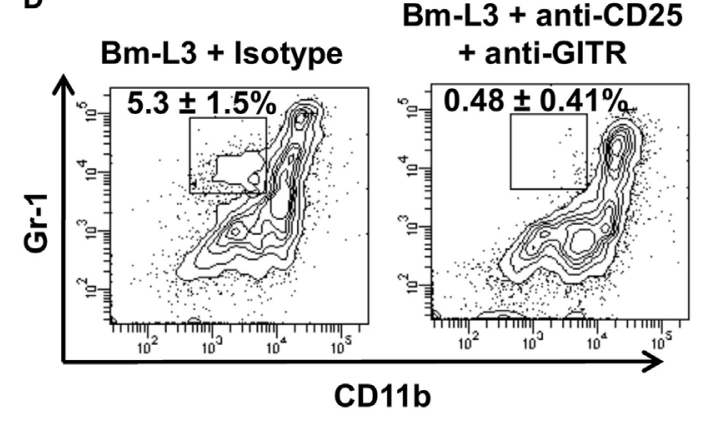

FIGURE 6 | Neutralization of regulatory T-cells affects the accumulation of other leukocyte subsets. Representative flow cytometry dot plots showing (A) granulocytes [low forward scatter (FSC)-A, high SSC-A], (B) eosinophils (Sig F+, MHCII-), (C) regulatory B cells (CD1 d $\mathrm{d}^{+}$, CD5a+), and (D) inflammatory monocytes $\left(\mathrm{Gr}^{+}{ }^{+}, \mathrm{CD} 11 \mathrm{~b}^{+}\right)$present in the spleens of anti-CD25 + anti-GITR-treated mice or uninfected lgG control at day 7 p.i. All values represent mean \pm SD values from three independent experiments with at least 3-4 animals/group.

contributed to reduced Bm-L3 burden, we reasoned if this treatment increased the longevity of M2 MФs by interfering with their apoptotic machinery. We, therefore, assessed the role of caspases and found reduced expression of caspase $3(p \leq 0.001)$, and caspase $6(p \leq 0.01)$ in the cell lysate of FACS-sorted splenic MФs from Bm-L3-infected mice that received anti-CD25 + antiGITR treatment as compared to those that received IgG isotypes (Figure 7). Notably, no change was observed in the activity of caspase 2, 8 , and 9, which not only suggested differential regulation of caspases during Bm-L3 infection, but also underlined reduced caspase activity as one of the reasons that might have prolonged survival of M2 MФs that lead to enhanced killing of infective larvae (16).

\section{DISCUSSION}

Filarial parasites dampen the host immune response by interfering with the cellular and molecular mechanisms involved in the host defense, including but not limited to interfering with the functional plasticity of host MФs (17-19). However, mechanisms that regulate the polarization of $M \Phi$ s and support the establishment and survival of the parasite within the host are largely unexplored. In the present study, we infected BALB/c mice with infective larval stage 3 of the filarial parasite Brugia malayi and studied the polarization of host $M \Phi$ s during the first week of infection. Early perturbations observed in the cytokine secreting pattern of splenic MФs suggested alternatively activated phenotype of MФs at day 3 post Bm-L3 infection, which gradually shifted to a regulatory phenotype by day 7 p.i. indicating that polarization of splenic MФs was rapid and took place within the first week of infection itself. In fact, TGF $\beta 1$, an antiinflammatory cytokine that is often listed in the same category as IL-10 was elevated at day 3 p.i., which might have aided in the generation of the M2 phenotype. Support for this notion is evident from the work done by Heisterkamp and colleagues who showed that TGF $\beta$ signaling was critical for promoting alternative macrophage activation (20).

Alternative metabolic state in murine M $\Phi$ s is reflected by the nitric oxide synthase/arginase balance that is correlated with Th1/Th2 phenotype $(21,22)$. While Ym-1 inhibits $12 / 15$-LOX signaling pathway that augments Th2 immunity (23-25), Fizz-1 is critically involved in wound repair, impaired worm clearance and inhibition of type 2 immunity that is suggestive of a regulatory phenotype (26). Our findings when seen in the context of Bm-L3 molting reveal an interesting scenario as Bm-L3 undergo their first molting around day 7 inside the mice and are thus highly exposed to the immune cells, so, it is in the interest of the parasite to manipulate the host immune system toward a regulatory phenotype so that no alarm signals are generated that may be harmful to the parasite. We, therefore, believe that the observed shift in the phenotype of host MФs from M2 to Mregs around day 7 p.i. is indeed a reflection of a cleverly mastered immune evasion strategy employed by the infective larvae to escape the host immune surveillance at the earliest host-parasite interface $(27,28)$. In this context, it is worth considering the work done by Edwards and colleagues who demonstrated tremendous plasticity 

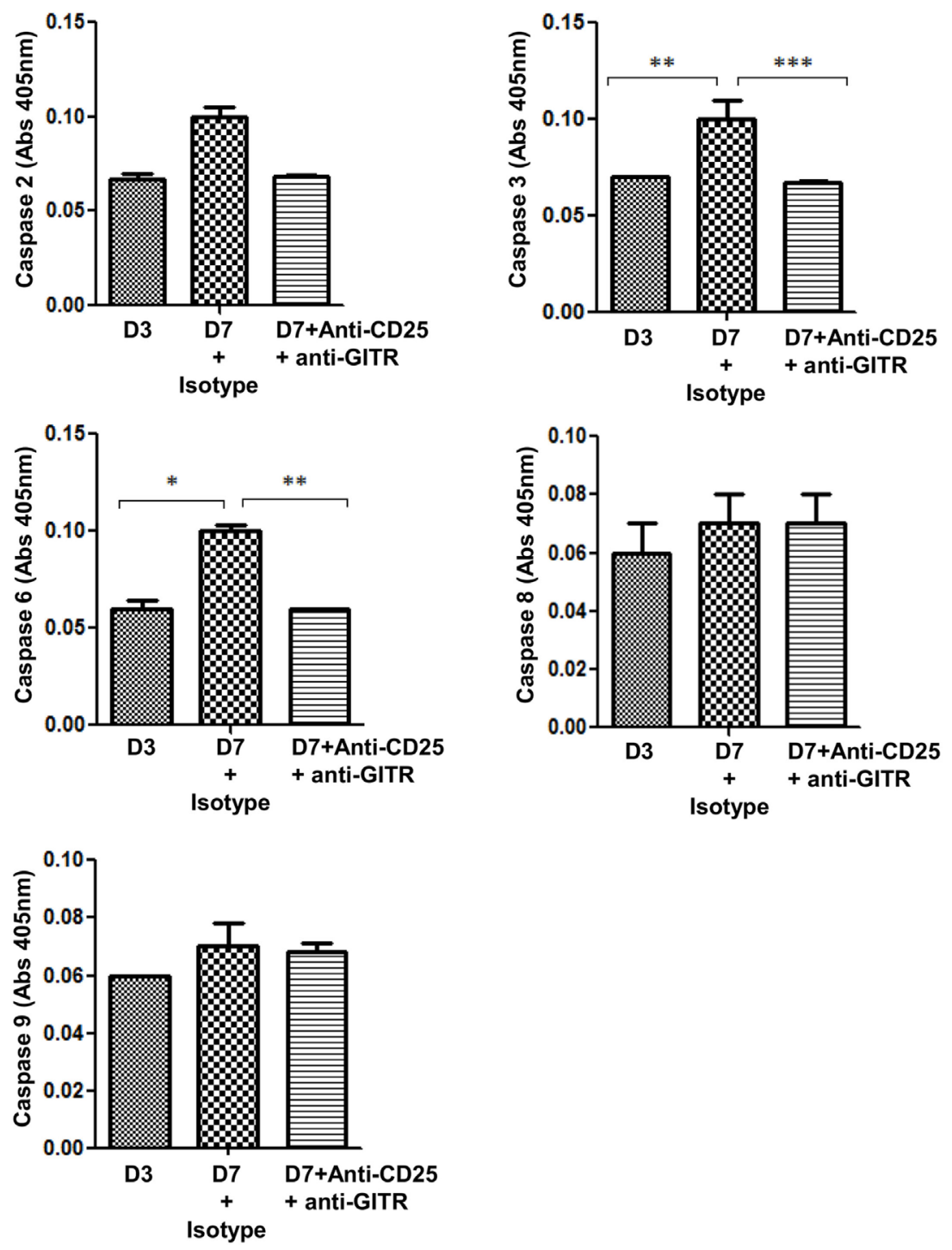

FIGURE 7 | Neutralization of regulatory T-cells delays apoptosis in M2 MФs. Bar graphs represent caspase activity in the lysate of FACS-sorted splenic MФs from anti-CD25 + anti-GITR-treated mice or uninfected lgG control at day 7 p.i. All values represent mean \pm SD values from two independent experiments with at least 3-4 animals/group. $p$-Value of $\leq 0.05, \leq 0.01$, and $\leq 0.001$ was considered significant, highly significant, and very highly significant and marked with ${ }^{*},{ }^{\star *}$, and ${ }^{* \star *}$, respectively.

of bone marrow-derived MФ (BMM $\Phi$ ) when primed with IFN- $\gamma$, LPS, immune complexes, or IL-4 (3). Our study and that of Edwards et al. thus demonstrate the highly plastic nature of these cells, which profoundly influence the outcome of an immune response $(29,30)$.

Elevated levels of maturation and co-stimulatory markers and reduced support for $\mathrm{T}$ cell proliferation strengthened the regulatory phenotype of $\mathrm{M} \Phi$ s at day 7 p.i., which may have been due to their suppressive nature and the presence of regulatory cytokines like IL-10 at day 7 p.i. $(31,32)$ or even ligation of PD-L1 to its receptor PD-1 on T cells as documented in previous reports (33-35). In fact, studies have also shown that engagement of PD-L1 with its receptor PD-1 on T cells not only delivers a signal that inhibits the proliferation of $\mathrm{T}$ cells but also inhibits
TCR-mediated IL-2 production (33-35). In addition to this, role of lipid mediators has also been documented in the suppression of T cell proliferation (36). Our observation of higher PGE2 levels corroborated with reports documenting that immune complex/Ig negatively regulate TLR4-triggered inflammatory response in MФs through Fc gamma RIIb-dependent PGE2 production (37) which is a selective inducer of IL-12p40 (38). Not only this, induction of PGE2 by microfilariae of Wuchereria bancrofti and Brugia malayi has been documented and is believed to play a role in the host-parasite communication (39). Also, PGE2 being a potent immunomodulatory lipid mediator has also been implicated in the survival of Trypanosoma cruzi within the host (40). Previous reports have also documented that PGE2 enhances type 2 immunity by upregulating the 
production of CCL22 (41). When coalesced together, these observations provide support to the notion that elevated levels of PGE2 acted as a key regulator behind the polarization of M2 MФs to Mregs (42).

Another notable observation of the present study was elevated expression level of TLR2 in host MФs following Bm-L3 infection. Previous reports have not only documented the role of TLR2 in the induction of Tregs by Schistosoma mansoni egg antigens but activation of TLR 2 by Schistosomal lysophosphatidylserine has been shown to affect immune polarization also $(43,44)$. In addition to this, numerous reports have demonstrated interaction between TLR $2 / 4$ to be responsible for the anti-inflammatory response induced by helminthderived products (45).

We also observed attenuated phosphorylation of $N F-\kappa B$, which is centrally involved in the regulation of inflammatory cytokines during infection and stress conditions $(46,47)$. Significantly upregulated levels of Erk1/2, but not that of p-p38 in $\mathrm{M} 2 \mathrm{M} \Phi$ s at day 3 p.i. suggested that $M \Phi$ polarization was mainly regulated by Erk1/2. Previous studies have demonstrated that ES-62, an excretory-secretory product of the rodent filarial nematode Acanthocheilonema viteae induced tyrosine phosphorylation of glycoproteins in murine macrophages (48) and modulated the activation of MAP kinases, thereby regulating cytokine production (49). In agreement with this data, our results also indicated significant upregulation of IL-4 when ERK activity was blocked, which checked the polarization of M2 MФs to Mregs.

The heightened PTPs activity and upregulated transcript levels of six major phosphatases further supported the reasoning that the process of macrophage polarization was indeed regulated at many levels. In fact, reports have suggested that conditional removal of Shp2 in monocytes/macrophages lead to an IL-4-mediated shift toward a M2 phenotype. Additionally, an increase in arginase activity was detected in $\operatorname{Shp} 2(\Delta / \Delta)$ mice after i.p. injection of chitin, whereas Shp2-deficient MФs showed enhanced M2 polarization and protection against schistosome egg-induced schistosomiasis (50).

Our observations of CCL22-mediated significant expansion of Tregs in the spleens of mice was corroborated with previous reports documenting the expansion of Tregs within 3-7 days of helminthes infection (51-54). As such, during chronic filarial infection in humans, Tregs secrete regulatory cytokines like CCL-4 that suppresses tumor-specific and inflammatory responses $(55,56)$. Also, Tregs from filariasis-infected individuals produce IL-29, a member of the IL-10 cytokine family, which exhibits antitumor and antiviral activities that programs naïve DCs to induce Treg differentiation (57, 58). Although Tregs are not a major source of IL-10 secretion during chronic human infections (59), a recent report showed that depletion of CD25 ${ }^{\text {hi }}$ cells significantly upregulated cytokine production and the proliferation of $\mathrm{B}$ and $\mathrm{T}$ lymphocytes during patent filarial infection, which suggested that Tregs from chronically infected filarial patients were functionally more suppressive (60).

Importantly, neutralization of Tregs activity by function blocking anti-CD25 + anti-GITR antibodies not only checked the polarization of M2 MФs to Mregs but also increased the percentages of eosinophils, which assisted in Bm-L3 killing (61). Notably, eosinophils have well-documented antihelminth properties and increased percentages of eosinophils after Treg neutralization has also been observed in cancer (62). Importantly, infection with Strongyloides stercoralis has been shown to induce alternatively activated macrophages within the peritoneal cavity of mice, which not only killed the worms in vivo but also collaborated with neutrophils and complement during the in vitro killing process (63). Furthermore, recent reports have also demonstrated that M2 MФ exhibit helminth killing activity in vivo provided the contact time between these $M \Phi$ s and the parasite is of sufficient duration, thereby implying that M2 MФs exert a protective role during parasitic infections as their depletion also inhibit worm expulsion (5). In fact, downregulated activity of some caspases in the anti-CD25 + anti-GITR-treated mice was an interesting observation that needs to be explored in greater detail in order to elucidate the contribution of caspases in the polarization and longevity of host MФs.

Taken together, the present study not only documents the multifarious and intriguingly complex nature of events that regulate the polarization of host $M \Phi$ s during the early phase of filarial infection (Figure 8) but also emphasizes on the hitherto unexplored cross talk between regulatory T-cells in the polarization of host MФs which help in the establishment and survival of the infective larvae inside the host. Identification of the parasite molecules that utilize the functional plasticity of MФs to subvert host immunity would be important for the development of effective therapeutic strategies that can halt or arrest the establishment of the filarial parasite at the earliest host-parasite interface.

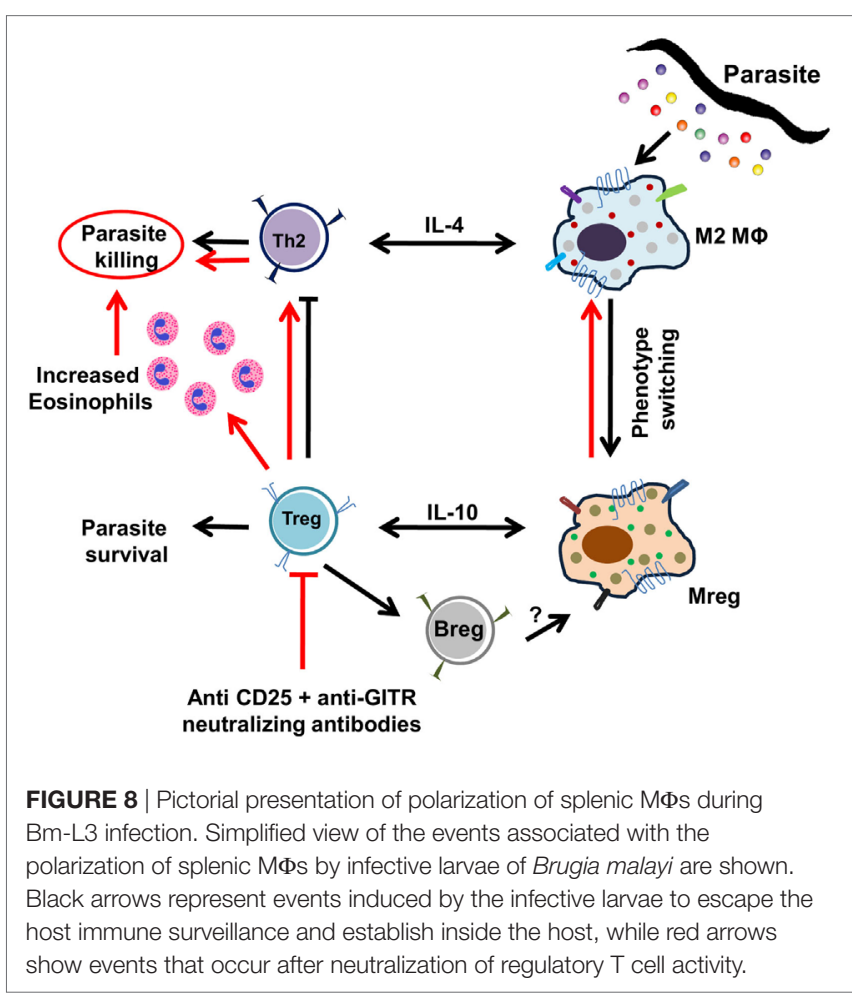




\section{ETHICS STATEMENT}

This study was carried out in accordance with the recommendations of Institutional Animal Ethics Committee. The protocol was approved by the Institutional Animal Ethics Committee.

\section{AUTHOR CONTRIBUTIONS}

AS and MS conceived and designed the experiments, analyzed the data, and wrote the manuscript. AS, PS, LG, and NS performed the experiments. MS and AV carried out cell sorting. SM helped in the maintenance of Brugia malayi infection and provided infective larvae. The authors thankfully acknowledge excellent technical support provided by O. P. Yadav for maintaining B. malayi infection in the laboratory.

\section{REFERENCES}

1. Dalton DK, Pitts-Meek S, Keshav S, Figari IS, Bradley A, Stewart TA. Multiple defects of immune cell function in mice with disrupted interferon-gamma genes. Science (1993) 259:1739-42. doi:10.1126/science.8456300

2. Gordon S. Alternative activation of macrophages. Nat Rev Immunol (2003) 3:23-35. doi:10.1038/nri978

3. Edwards JP, Zhang X, Frauwirth KA, Mosser DM. Biochemical and functional characterization of three activated macrophage populations. J Leukoc Biol (2006) 80:1298-307. doi:10.1189/jlb.0406249

4. Allen JE, Loke P. Divergent roles for macrophages in lymphatic filariasis. Parasite Immunol (2001) 23:345-52. doi:10.1046/j.1365-3024.2001.00394.x

5. Anthony RM, Urban JF Jr, Alem F, Hamed HA, Rozo CT, Boucher JL, et al. Memory $\mathrm{T}(\mathrm{H}) 2$ cells induce alternatively activated macrophages to mediate protection against nematode parasites. Nat Med (2006) 12:955-60. doi: $10.1038 / \mathrm{nm} 1451$

6. Sharma A. Functional impairment of murine dendritic cell subsets following infection with infective larval stage 3 of Brugia malayi. Infect Immun (2017) 85(1):e00818-16. doi:10.1128/IAI.00818-16

7. Sharma P, Sharma A, Vishwakarma AL, Agnihotri PK, Sharma S, Srivastava M. Host lung immunity is severely compromised during tropical pulmonary eosinophilia: role of lung eosinophils and macrophages. J Leukoc Biol (2015) 99(4):619-28. doi:10.1189/jlb.4A0715-309RR

8. Srivastava M, Meinders A, Steinwede K, Maus R, Lucke N, Buhling F, et al. Mediator responses of alveolar macrophages and kinetics of mononuclear phagocyte subset recruitment during acute primary and secondary mycobacterial infections in the lungs of mice. Cell Microbiol (2007) 9:738-52. doi:10.1111/j.1462-5822.2006.00824.x

9. Pathak M, Verma M, Srivastava M, Misra-Bhattacharya S. Wolbachia endosymbiont of Brugia malayi elicits a T helper type 17-mediated pro-inflammatory immune response through Wolbachia surface protein. Immunology (2015) 144:231-44. doi:10.1111/imm.12364

10. Srivastava M, Jung S, Wilhelm J, Fink L, Buhling F, Welte T, et al. The inflammatory versus constitutive trafficking of mononuclear phagocytes into the alveolar space of mice is associated with drastic changes in their gene expression profiles. J Immunol (2005) 175:1884-93. doi:10.4049/jimmunol. 175.3.1884

11. Livak KJ, Schmittgen TD. Analysis of relative gene expression data using realtime quantitative PCR and the 2(-delta delta C(T)) method. Methods (2001) 25:402-8. doi:10.1006/meth.2001.1262

12. Everts B, Adegnika AA, Kruize YC, Smits HH, Kremsner PG, Yazdanbakhsh M. Functional impairment of human myeloid dendritic cells during Schistosoma haematobium infection. PLoS Negl Trop Dis (2010) 4:e667. doi:10.1371/ journal.pntd.0000667

13. Fordham-Skelton AP, Chilley P, Lumbreras V, Reignoux S, Fenton TR, Dahm CC, et al. A novel higher plant protein tyrosine phosphatase interacts with SNF1related protein kinases via a KIS (kinase interaction sequence) domain. Plant $J$ (2002) 29:705-15. doi:10.1046/j.1365-313X.2002.01250.x

\section{FUNDING}

PS, LG, NS, and AS thankfully acknowledge fellowship support from University Grants Commission (UGC) and Council of Scientific and Industrial Research (CSIR), New Delhi respectively. This work was supported by DST-SERB Grant No. EMR/2016/001880 and grants provided under CSIRNetwork projects "New approaches toward understanding of Disease dynamics and to accelerate drug discovery (UNDO)" and "Emerging and re-emerging challenges in infectious diseases: Systems based drug design for infectious diseases (SPLenDID)" to MS. The funders had no role in study design, data collection, analysis, decision to publish, or preparation of the manuscript. This is communication number 9624 from CSIR-CDRI.

14. Pathak M, Sharma P, Sharma A, Verma M, Srivastava M, Misra-Bhattacharya S Regulatory T-cell neutralization in mice during filariasis helps in parasite clearance by enhancing $\mathrm{T}$ helper type 17-mediated pro-inflammatory response. Immunology (2016) 147:190-203. doi:10.1111/imm.12550

15. Ekman P, Jager O. Quantification of subnanomolar amounts of phosphate bound to seryl and threonyl residues in phosphoproteins using alkaline hydrolysis and malachite green. Anal Biochem (1993) 214:138-41. doi:10.1006/ abio. 1993.1468

16. Yao Y, Shi Q, Chen B, Wang Q, Li X, Li L, et al. Identification of caspase-6 as a new regulator of alternatively activated macrophages. J Biol Chem (2016) 291:17450-66. doi:10.1074/jbc.M116.717868

17. O’Regan NL, Steinfelder S, Venugopal G, Rao GB, Lucius R, Srikantam A, et al. Brugia malayi microfilariae induce a regulatory monocyte/macrophage phenotype that suppresses innate and adaptive immune responses. PLoS Negl Trop Dis (2014) 8:e3206. doi:10.1371/journal.pntd.0003206

18. Nasarre C, Krahenbuhl JL, Klei TR. Down regulation of macrophage activation in Brugia pahangi-infected jirds (Meriones unguiculatus). Infect Immun (1998) 66:1063-9.

19. Stout RD, Suttles J. Functional plasticity of macrophages: reversible adaptation to changing microenvironments. J Leukoc Biol (2004) 76:509-13. doi:10.1189/ jlb.0504272

20. Gong D, Shi W, Yi SJ, Chen H, Groffen J, Heisterkamp N. TGFbeta signaling plays a critical role in promoting alternative macrophage activation. BMC Immunol (2012) 13:31. doi:10.1186/1471-2172-13-31

21. Munder M, Eichmann K, Modolell M. Alternative metabolic states in murine macrophages reflected by the nitric oxide synthase/arginase balance: competitive regulation by CD4+ T cells correlates with Th1/Th2 phenotype. J Immunol (1998) 160:5347-54.

22. Iniesta V, Gomez-Nieto LC, Molano I, Mohedano A, Carcelen J, Miron C, et al. Arginase I induction in macrophages, triggered by Th2-type cytokines, supports the growth of intracellular Leishmania parasites. Parasite Immunol (2002) 24:113-8. doi:10.1046/j.1365-3024.2002.00444.x

23. Cai Y, Kumar RK, Zhou J, Foster PS, Webb DC. Ym1/2 promotes Th 2 cytokine expression by inhibiting 12/15(S)-lipoxygenase: identification of a novel pathway for regulating allergic inflammation. J Immunol (2009) 182:5393-9. doi:10.4049/jimmunol.0803874

24. Owhashi M, Arita H, Hayai N. Identification of a novel eosinophil chemotactic cytokine (ECF-L) as a chitinase family protein. J Biol Chem (2000) 275:1279-86. doi:10.1074/jbc.275.2.1279

25. Nair MG, Cochrane DW, Allen JE. Macrophages in chronic type 2 inflammation have a novel phenotype characterized by the abundant expression of Ym1 and Fizzl that can be partly replicated in vitro. Immunol Lett (2003) 85:173-80. doi:10.1016/S0165-2478(02)00225-0

26. Pesce JT, Ramalingam TR, Wilson MS, Mentink-Kane MM, Thompson RW, Cheever AW, et al. Retnla (relmalpha/fizz1) suppresses helminth-induced Th2type immunity. PLoS Pathog (2009) 5:e1000393. doi:10.1371/journal.ppat.1000393

27. ReyesJL, TerrazasCA,Alonso-TrujilloJ,van RooijenN,SatoskarAR, TerrazasLI. Early removal of alternatively activated macrophages leads to Taenia crassiceps 
cysticercosis clearance in vivo. Int J Parasitol (2010) 40:731-42. doi:10.1016/j. ijpara.2009.11.014

28. Kropf P, Fuentes JM, Fahnrich E, Arpa L, Herath S, Weber V, et al. Arginase and polyamine synthesis are key factors in the regulation of experimental leishmaniasis in vivo. FASEB J (2005) 19:1000-2. doi:10.1096/fj.04-3416fje

29. Mosser DM. The many faces of macrophage activation. J Leukoc Biol (2003) 73:209-12. doi:10.1189/jlb.0602325

30. Hume DA. The many alternative faces of macrophage activation. Front Immunol (2015) 6:370. doi:10.3389/fimmu.2015.00370

31. Hesse M, Piccirillo CA, Belkaid Y, Prufer J, Mentink-Kane M, Leusink M, et al. The pathogenesis of schistosomiasis is controlled by cooperating IL-10-producing innate effector and regulatory T cells. J Immunol (2004) 172:3157-66. doi:10.4049/jimmunol.172.5.3157

32. Osborne J, Devaney E. Interleukin-10 and antigen-presenting cells actively suppress Th1 cells in BALB/c mice infected with the filarial parasite Brugia pahangi. Infect Immun (1999) 67:1599-605.

33. Karwacz K, Bricogne C, MacDonald D, Arce F, Bennett CL, Collins M, et al. PD-L1 co-stimulation contributes to ligand-induced T cell receptor down-modulation on CD8+ T cells. EMBO Mol Med (2011) 3:581-92. doi:10.1002/emmm.201100165

34. Terrazas LI, Montero D, Terrazas CA, Reyes JL, Rodriguez-Sosa M. Role of the programmed death-1 pathway in the suppressive activity of alternatively activated macrophages in experimental cysticercosis. Int J Parasitol (2005) 35:1349-58. doi:10.1016/j.ijpara.2005.06.003

35. Smith P, Walsh CM, Mangan NE, Fallon RE, Sayers JR, McKenzie AN, et al. Schistosoma mansoni worms induce anergy of T cells via selective upregulation of programmed death ligand 1 on macrophages. J Immunol (2004) 173:1240-8. doi:10.4049/jimmunol.173.2.1240

36. Brys L, Beschin A, Raes G, Ghassabeh GH, Noel W, Brandt J, et al. Reactive oxygen species and 12/15-lipoxygenase contribute to the antiproliferative capacity of alternatively activated myeloid cells elicited during helminth infection. J Immunol (2005) 174:6095-104. doi:10.4049/jimmunol.174.10.6095

37. Zhang Y, Liu S, Liu J, Zhang T, Shen Q, Yu Y, et al. Immune complex/Ig negatively regulate TLR4-triggered inflammatory response in macrophages through Fc gamma RIIb-dependent PGE2 production. JImmunol (2009) 182:554-62. doi:10.4049/jimmunol.182.1.554

38. Kalinski P, Vieira PL, Schuitemaker JH, de Jong EC, Kapsenberg ML. Prostaglandin E(2) is a selective inducer of interleukin-12 p40 (IL-12p40) production and an inhibitor of bioactive IL-12p70 heterodimer. Blood (2001) 97:3466-9. doi:10.1182/blood.V97.11.3466

39. Liu LX, Buhlmann JE, Weller PF. Release of prostaglandin E2 by microfilariae of Wuchereria bancrofti and Brugia malayi. Am J Trop Med Hyg (1992) 46:520-3. doi:10.4269/ajtmh.1992.46.520

40. Toledo DA, Roque NR, Teixeira L, Milan-Garces EA, Carneiro AB, Almeida MR, et al. Lipid body organelles within the parasite Trypanosoma cruzi: a role for intracellular arachidonic acid metabolism. PLoS One (2016) 11:e0160433. doi:10.1371/journal.pone.0160433

41. Kuroda E, Sugiura T, Okada K, Zeki K, Yamashita U. Prostaglandin E2 up-regulates macrophage-derived chemokine production but suppresses IFN-inducible protein-10 production by APC. J Immunol (2001) 166:1650-8. doi:10.4049/jimmunol.166.3.1650

42. Dioszeghy V, Rosas M, Maskrey BH, Colmont C, Topley N, Chaitidis P, et al. 12/15-lipoxygenase regulates the inflammatory response to bacterial products in vivo. J Immunol (2008) 181:6514-24. doi:10.4049/jimmunol.181.9.6514

43. van der Kleij D, Latz E, Brouwers JF, Kruize YC, Schmitz M, Kurt-Jones EA, et al. A novel host-parasite lipid cross-talk. Schistosomal lyso-phosphatidylserine activates toll-like receptor 2 and affects immune polarization. J Biol Chem (2002) 277:48122-9. doi:10.1074/jbc.M206941200

44. Panther E, Idzko M, Corinti S, Ferrari D, Herouy Y, Mockenhaupt M, et al. The influence of lysophosphatidic acid on the functions of human dendritic cells. J Immunol (2002) 169:4129-35. doi:10.4049/jimmunol.169.8.4129

45. van Riet E, Everts B, Retra K, Phylipsen M, van Hellemond JJ, Tielens AG, et al. Combined TLR2 and TLR4 ligation in the context of bacterial or helminth extracts in human monocyte derived dendritic cells: molecular correlates for Th1/Th2 polarization. BMCImmunol (2009) 10:9. doi:10.1186/1471-2172-10-9

46. Mancino A, Lawrence T. Nuclear factor-kappaB and tumor-associated macrophages. Clin Cancer Res (2010) 16:784-9. doi:10.1158/1078-0432.CCR-09-1015

47. Baker RG, Hayden MS, Ghosh S. NF-kappaB, inflammation, and metabolic disease. Cell Metab (2011) 13:11-22. doi:10.1016/j.cmet.2010.12.008
48. Goodridge HS, Deehan MR, Harnett W, Harnett MM. Subversion of immunological signalling by a filarial nematode phosphorylcholine-containing secreted product. Cell Signal (2005) 17:11-6. doi:10.1016/j.cellsig.2004.05.014

49. Goodridge HS, Harnett W, Liew FY, Harnett MM. Differential regulation of interleukin-12 p40 and p35 induction via Erk mitogen-activated protein kinase-dependent and -independent mechanisms and the implications for bioactive IL-12 and IL-23 responses. Immunology (2003) 109:415-25. doi:10.1046/j.1365-2567.2003.01689.x

50. Tao B, Jin W, Xu J, Liang Z, Yao J, Zhang Y, et al. Myeloid-specific disruption of tyrosine phosphatase Shp2 promotes alternative activation of macrophages and predisposes mice to pulmonary fibrosis. J Immunol (2014) 193:2801-11. doi:10.4049/jimmunol.1303463

51. Taylor MD, van der Werf N, Harris A, Graham AL, Bain O, Allen JE, et al. Early recruitment of natural CD4+ Foxp3+ Treg cells by infective larvae determines the outcome of filarial infection. Eur J Immunol (2009) 39:192-206. doi:10.1002/eji.200838727

52. Blankenhaus B, Klemm U, Eschbach ML, Sparwasser T, Huehn J, Kuhl AA, et al. Strongyloides ratti infection induces expansion of Foxp3+ regulatory $\mathrm{T}$ cells that interfere with immune response and parasite clearance in $\mathrm{BALB} / \mathrm{c}$ mice. J Immunol (2011) 186:4295-305. doi:10.4049/jimmunol.1001920

53. Liu G, Ma H, Qiu L, Li L, Cao Y, Ma J, et al. Phenotypic and functional switch of macrophages induced by regulatory $\mathrm{CD} 4+\mathrm{CD} 25+\mathrm{T}$ cells in mice. Immunol Cell Biol (2011) 89:130-42. doi:10.1038/icb.2010.70

54. D'Elia R, Behnke JM, Bradley JE, Else KJ. Regulatory T cells: a role in the control of helminth-driven intestinal pathology and worm survival. J Immunol (2009) 182:2340-8. doi:10.4049/jimmunol.0802767

55. Joosten SA, van Meijgaarden KE, Savage ND, de Boer T, Triebel F, van der Wal A, et al. Identification of a human CD8+ regulatory $\mathrm{T}$ cell subset that mediates suppression through the chemokine CC chemokine ligand 4. Proc Natl Acad Sci U S A (2007) 104:8029-34. doi:10.1073/pnas.0702257104

56. Metenou S, Coulibaly YI, Sturdevant D, Dolo H, Diallo AA, Soumaoro L, et al. Highly heterogeneous, activated, and short-lived regulatory $\mathrm{T}$ cells during chronic filarial infection. Eur J Immunol (2014) 44:2036-47. doi:10.1002/eji.201444452

57. Commins S, Steinke JW, Borish L. The extended IL-10 superfamily: IL-10, IL-19, IL-20, IL-22, IL-24, IL-26, IL-28, and IL-29. J Allergy Clin Immunol (2008) 121:1108-11. doi:10.1016/j.jaci.2008.02.026

58. Witte K, Witte E, Sabat R, Wolk K. IL-28A, IL-28B, and IL-29: promising cytokines with type I interferon-like properties. Cytokine Growth Factor Rev (2010) 21:237-51. doi:10.1016/j.cytogfr.2010.04.002

59. Mitre E, Chien D, Nutman TB. CD4(+) (and not CD25+) T cells are the predominant interleukin-10-producing cells in the circulation of filaria-infected patients. J Infect Dis (2008) 197:94-101. doi:10.1086/524301

60. Wammes LJ, Hamid F, Wiria AE, Wibowo H, Sartono E, Maizels RM, et al. Regulatory T cells in human lymphatic filariasis: stronger functional activity in microfilaremics. PLoS Negl Trop Dis (2012) 6:e1655. doi:10.1371/journal. pntd.0001655

61. Mylonas KJ, Nair MG, Prieto-Lafuente L, Paape D, Allen JE. Alternatively activated macrophages elicited by helminth infection can be reprogrammed to enablemicrobialkilling.J Immunol(2009) 182:3084-94.doi:10.4049/jimmunol. 0803463

62. Carretero R, Sektioglu IM, Garbi N, Salgado OC, Beckhove P, Hammerling GJ. Corrigendum: eosinophils orchestrate cancer rejection by normalizing tumor vessels and enhancing infiltration of CD8(+) T cells. Nat Immunol (2016) 17:214. doi:10.1038/ni0216-214b

63. Bonne-Annee S, Kerepesi LA, Hess JA, O'Connell AE, Lok JB, Nolan TJ, et al. Human and mouse macrophages collaborate with neutrophils to kill larval Strongyloides stercoralis. Infect Immun (2013) 81:3346-55. doi:10.1128/IAI.00625-13

Conflict of Interest Statement: The authors declare that the research was conducted in the absence of any commercial or financial relationships that could be construed as a potential conflict of interest.

Copyright (c) 2018 Sharma, Sharma, Ganga, Satoeya, Mishra, Vishwakarma and Srivastava. This is an open-access article distributed under the terms of the Creative Commons Attribution License (CC BY). The use, distribution or reproduction in other forums is permitted, provided the original author(s) and the copyright owner are credited and that the original publication in this journal is cited, in accordance with accepted academic practice. No use, distribution or reproduction is permitted which does not comply with these terms. 